\title{
Autoreactive helper T cells alleviate the need for intrinsic TLR signaling in autoreactive B cell activation
}

\author{
Josephine R. Giles,, ${ }^{1,2}$ Adriana Turqueti Neves, ${ }^{2}$ Ann Marshak-Rothstein, ${ }^{3}$ and Mark J. Shlomchik ${ }^{1,2}$ \\ 'Departments of Laboratory Medicine and Immunobiology, Yale University School of Medicine, New Haven, Connecticut, \\ USA. Department of Immunology, University of Pittsburgh School of Medicine, Pittsburgh, Pennsylvania, USA. \\ ${ }^{3}$ Division of Rheumatology, Department of Medicine, University of Massachusetts School of Medicine, \\ Worcester, Massachusetts, USA.
}

T cells play a significant role in the pathogenesis of systemic autoimmune diseases, including systemic lupus erythematosus; however, there is relatively little information on the nature and specificity of autoreactive T cells. Identifying such cells has been technically difficult because they are likely to be rare and low affinity. Here, we report a method for identifying autoreactive T cell clones that recognize proteins contained in autoantibody immune complexes, providing direct evidence that functional autoreactive helper $\mathrm{T}$ cells exist in the periphery of normal mice. These T cells significantly enhanced autoreactive B cell proliferation and altered B cell differentiation in vivo. Most importantly, these autoreactive T cells were able to rescue many aspects of the TLR-deficient AM14 (anti-IgC2a rheumatoid factor) B cell response, suggesting that TLR requirements can be bypassed. This result has implications for the efficacy of TLRtargeted therapy in the treatment of ongoing disease.

Conflict of interest: The authors have declared that no conflict of interest exists.

Submitted: September 23, 2016

Accepted: January 6, 2017

Published: February 23, 2017

Reference information:

JCI Insight. 2017;2(4):e90870. https:// doi.org/10.1172/jii.insight.90870.

\section{Introduction}

Systemic lupus erythematosus (SLE) was originally thought to be an Ab-mediated disease; however, the importance of $\mathrm{Ab}$-independent $\mathrm{B}$ cell functions and cellular immunity has now been recognized. While $\mathrm{B}$ cell receptor (BCR) transgenic model systems specific for lupus autoantigens (autoAgs) have elucidated many aspects of autoreactive B cell tolerance and pathogenicity $(1,2)$, much less is known about the identity, fate, and function of autoreactive $\mathrm{T}$ cells in systemic autoimmunity.

Self-reactive $\mathrm{T}$ cells have been identified in the repertoires of both healthy and autoimmune people and mice - suggesting that pathogenic specificities exist in a normal $\mathrm{T}$ cell repertoire but require the appropriate genetic background and environment for activation (3-5). However, detecting and assessing these cells is challenging due to thymic deletion of the highest affinity anti-self T cells as well as T cell anergy. Furthermore, unlike organ-specific autoimmunity, the identity of relevant autoAgs in systemic autoimmunity is less clear.

All studies to date have used a candidate approach to isolate autoreactive T cells, which has not allowed for the discovery of previously unknown specificities. Autoreactive T cells have been isolated using this candidate approach in SLE $(3,4,6)$, rheumatoid arthritis (7), mixed connective tissue disease (8), and diabetes (9). These studies used a specific peptide or protein that was already known to stimulate autoreactive $\mathrm{T}$ cells, such as insulin in the case of diabetes, or used a known B cell antigen (Ag); therefore, providing a very narrow view into the functional autoreactive $\mathrm{T}$ cell repertoire. There has been no method to isolate or study a more diverse population of antigen specific autoreactive T cells. This is critical in SLE and other systemic autoimmune diseases because the target $\mathrm{T}$ cell antigens are not well defined. This limited understanding of the T cell's role in systemic autoimmunity impedes proper understanding of the basic biology of these diseases, as well as developing better therapies.

To help bridge this critical gap in knowledge, we developed a method to identify autoreactive $\mathrm{T}$ cells without choosing a specific candidate antigen; we then used these T cells to study the T-B interactions that are central to SLE pathogenesis. Our strategy had two key components: first, it used IgG2a-specific AM14 rheumatoid factor (RF) B cells as antigen-presenting cells (APCs), and second, it used immune complexes (ICs) formed from authentic lupus autoantibodies (autoAbs) as Ag (10). As we show, by using AM14 B 
cells, we could stimulate self-reactive T cells without any a priori knowledge of the self-Ag or T cell specificity, other than that the self-Ag would be contained in the material targeted by bona fide lupus autoAbs.

AM14 B cells are quiescent but not tolerized ("ignorant") in normal animals but are activated by nucleic acid-containing ICs in vitro and in vivo (11). Since AM14 B cells do not become spontaneously activated in vitro in the absence of nucleic acid-containing ICs, we could establish Ag-free conditions, which is not possible typically when stimulating self-specific cells (e.g., if we had used a DNA-reactive B cell).

Stimulatory ICs are composed of an autoAb, such as antichromatin IgG2a, complexed with cellular debris from dying cells. BCR engagement of these ICs leads to internalization and delivery to MHC class II-processing compartments - enabling presentation of proteins within the IC (12). While autoAb-containing ICs are the target antigen in this system, they contain hundreds of self-proteins that may activate autoreactive T cells - allowing for the isolation of previously undiscovered $\mathrm{T}$ cell specificities.

The use of B cells as APCs is physiologically relevant, as we recently demonstrated a nonredundant role for B cells in activating T cells in the lupus-prone strain, MRL.Fas ${ }^{l p r}(13)$. Furthermore, B cells are far more potent APCs for their particular antigen compared with myeloid cells, which gives them the enhanced ability to activate low-affinity $\mathrm{T}$ cells, which is particularly salient in the search for autoreactive $\mathrm{T}$ cells that have escaped central deletion (14-17). RF B cells, which are found in multiple systemic autoimmune diseases, are unique in their ability to interactive with large and diverse population of $\mathrm{T}$ cells (Figure 1A). They can present self-Ags contained within ICs, which can be formed from any IgG2a Ab: anti-DNA, anti-RNA, anti-histone, anti-Sm, etc. This unique capacity for T cell activation may give RF $\mathrm{B}$ cells a pivotal role in epitope spreading and disease pathogenesis.

We chose to clone T cells from BALB/c mice, as we wanted to interrogate all potential precursors rather than just those that were highly selected at the end stage of an autoimmune process in lupusprone mice. Previous work in the lab indicated that cognate helper T cells for AM14 B cells exist in the repertoire of BALB/c mice (18).

Here, we report the identification of self-reactive $\mathrm{T}$ cell clones isolated with this approach and the in-depth characterization of two of them. We show how autoreactive $\mathrm{T}$ and $\mathrm{B}$ cells differentiate and interact upon stimulation with physiologic self-Ag. We further investigate how TLR and T cell-mediated signals interact and compensate for each other, with respect to both $\mathrm{B}$ cell and subsequent $\mathrm{T}$ cell activation. These studies also have important implications for lupus therapeutic intervention by TLR inhibition, with respect to whether TLR signaling is required to sustain as well as initiate systemic autoimmunity.

\section{Results}

Generation of IC-specific T cell lines. To activate and increase the frequency of potential IC-specific T cells, we cultured RF B cells from AM14 Vk8R BALB/c mice (hereafter referred to as AM14 B cells) with CD4 T cells from BALB/c mice in the presence of purified autoAbs (Figure 1, A and B). We used PL2-3, an antichromatin Ab that stimulates AM14 B cells in a TLR7- and TLR9-dependent manner (19), or Y2, an anti-Sm Ab precomplexed with Sm, which stimulates via TLR7 (20). Three days after the third round of stimulation, the cells were fused with BW5147 cells to generate T cell hybridomas, which were tested for reactivity to PL2-3 or Y2:Sm. We selected clones that did not respond to stimulation with AM14 B cells alone, AM14 B cell plus anti-IgM, and T-depleted splenocytes (SP APCs) alone (data not shown). The T cells were also tested in medium containing mouse serum to rule out bovine-specific reactivity (data not shown). We then sequenced the $\mathrm{T}$ cell receptor (TCR) genes and performed at least 2 rounds of limiting dilution, establishing unique clonal lines (Supplemental Table 1; supplemental material available online with this article; https://doi.org/10.1172/jci. insight.90870DS1): 5 T cell lines from 2 fusions from the PL2-3 stimulation cultures (of 960 hybridomas) and $3 \mathrm{~T}$ cell lines from 1 fusion from the Y2:Sm stimulation cultures (of 360 hybridomas).

In order to test whether the $\mathrm{T}$ cell epitope was from a protein contained within the IC, such as a DNA- or RNA-binding protein, as opposed to a peptide from PL2-3 or Y2, we stimulated the T cell clones with a panel of autoAbs of related specificities that originated from multiple mouse strains and that are composed of a variety gene segments (Figure 1, C and D, Supplemental Table 2). One clone, $13 \mathrm{C} 2$, responded to several autoAbs, including other antichromatin autoAbs and the anti-RNA autoAb, BWR4 (Figure 1C). Another clone, 1B9, responded to BWR4 and Ha310 in addition to PL2-3 (Figure 1C). These data strongly suggest that $13 \mathrm{C} 2$ and $1 \mathrm{~B} 9$ each recognize Ags that are bound by multiple autoAbs. The ability of multiple Abs to capture the antigenic proteins recognized by the same $\mathrm{T}$ cell clones is to be expected because these autoAbs bind to debris released from dying cells that would 
A

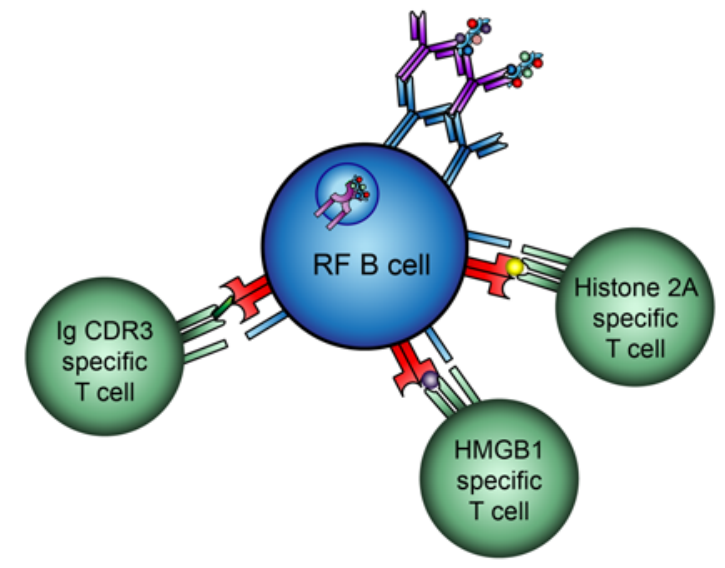

C
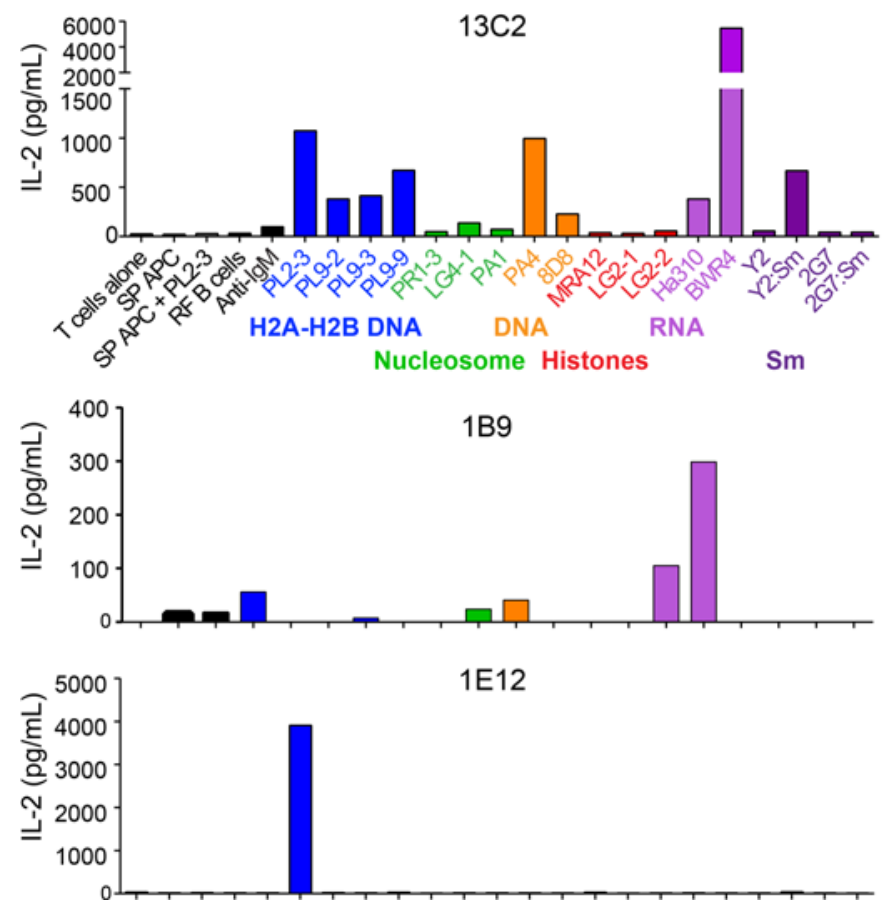

B

D
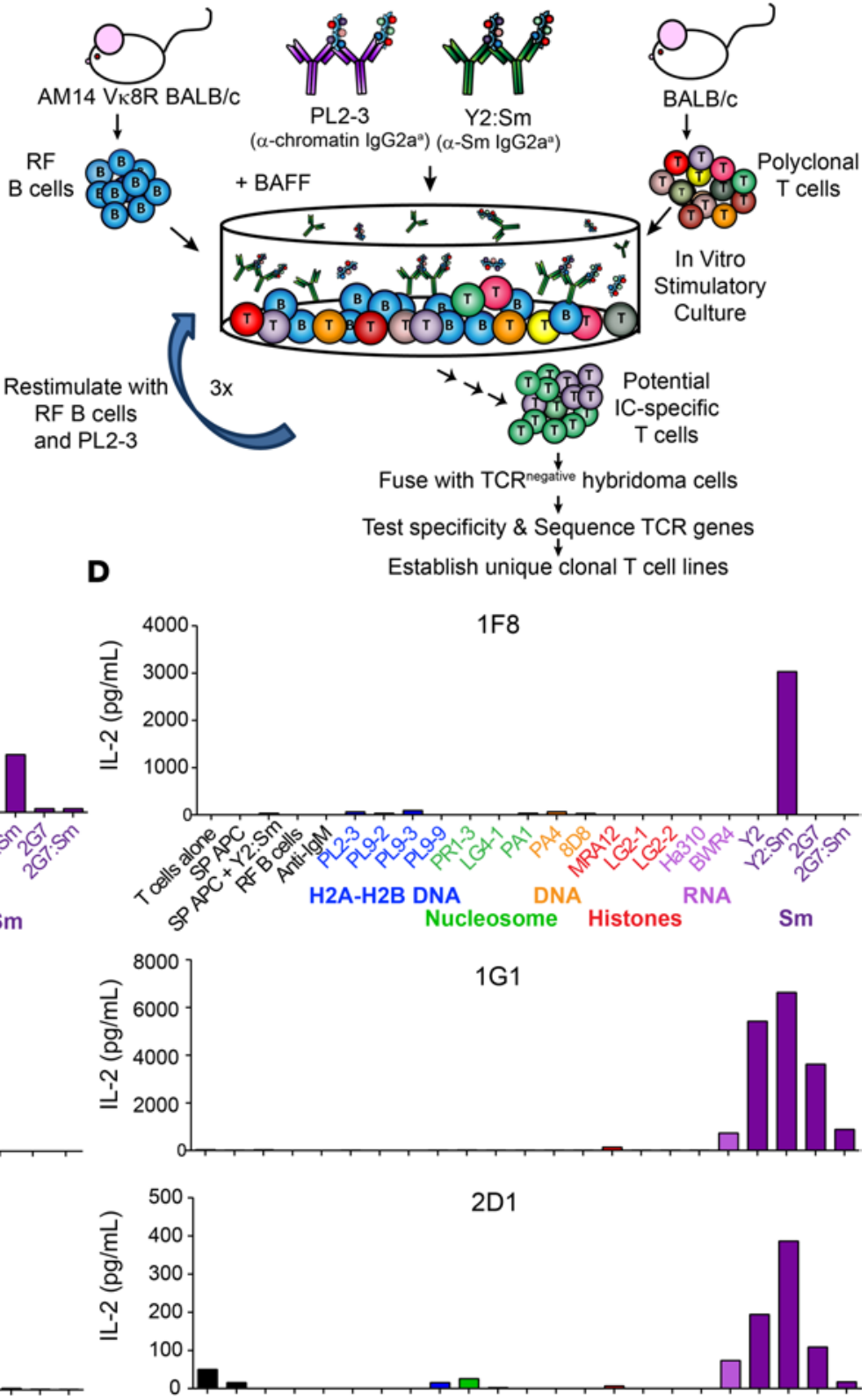

Figure 1. Generation of IC-specific T cells using AM14 B cells and autoAb ICs. (A) RF B cells have the potential to interact with a large repertoire of T cell specificities due to the complexity of autoAb ICs. (B) Experimental strategy. Hybridoma T cell lines originally stimulated with PL2-3 (C) or Y2:Sm (D) were cultured with SP APC or AM14 B cells in the presence the indicated Ab. IL-2 in the culture supernatant was measured 18 hours later by ELISA. Each graph is representative of at least 2 independent experiments.

include a complex mix of nucleic acids and associated proteins. Conversely, because the stimulatory Abs had diverse $\mathrm{V}$ region sequences that were also used by nonstimulatory autoAbs, it is highly unlikely that the $\mathrm{T}$ cell clones reacted with an Ig-derived peptide (Supplemental Table 2).

In contrast, $3 \mathrm{~T}$ cell clones responded only to PL2-3, of which the clone E12 is shown (Figure 1C). These clones may recognize peptides from Ags that are uniquely captured by PL2-3 but could also recognize $\mathrm{V}$ region peptides from the stimulating IgG2a; we chose to not continue with these clones, as they were likely to be of less general importance. From the Y2:Sm stimulations, one clone, 1F8, exclusively recognized the Y2:Sm complex (Figure 1D), suggesting that 1F8 is specific for an Ag that can only be bound by Y2. In contrast, $1 \mathrm{G} 1$ and $2 \mathrm{D} 1$ reacted to both $\mathrm{Y} 2$ and $2 \mathrm{G} 7$ and did not require the Abs to be precomplexed with Sm (Figure 1D), suggesting that they recognize an Ag released from dying cells in the culture.

To investigate whether the chromatin-specific $\mathrm{T}$ cells were relevant to in vivo disease, we tested whether these $\mathrm{T}$ cells could respond to AM14 B cells provided with autoAg ICs present in sera from diseased lupus- 
A

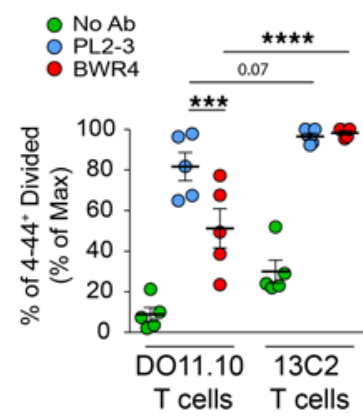

B
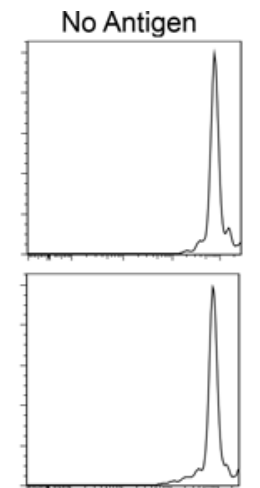

PL2-3
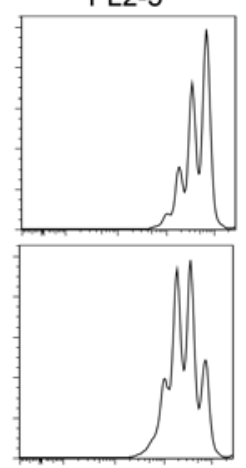

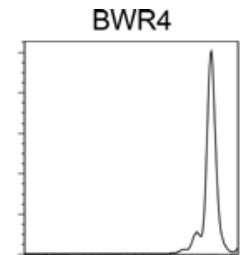

AM14 B cells

+ DO11.10

T cells

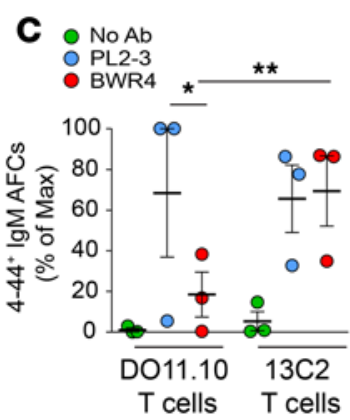

G

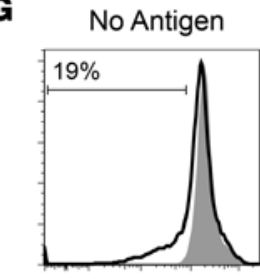

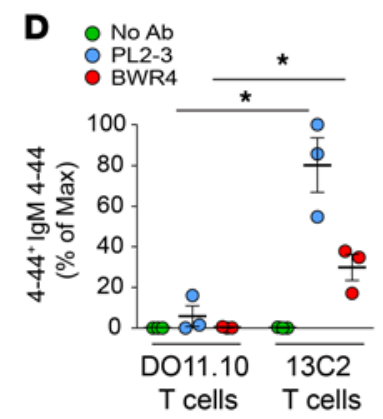

PL2-3

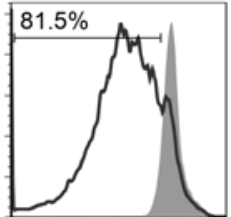

VPD450
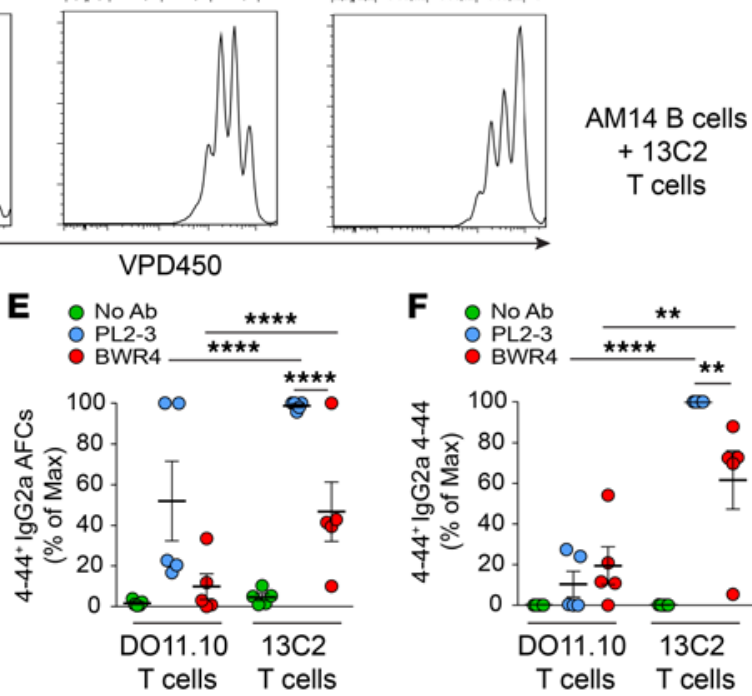

H

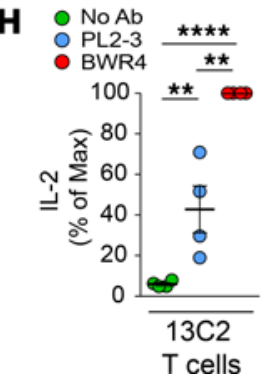

$\mathrm{T}$ cells
BWR4
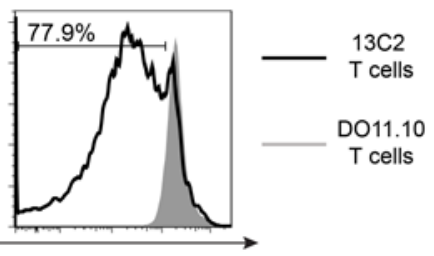

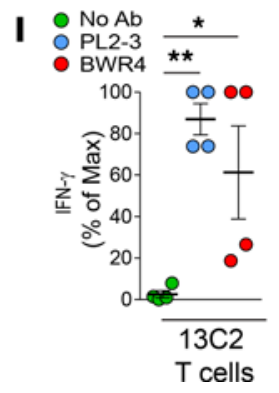

Figure 2. Primary IC-specific T cells respond to autoAb ICs and enhance AM14 B cell proliferation and differentiation in vitro. AM14 B cells were cultured with T cells from D011.10 BALB/c mice or from 13C2 Rg BALB/c mice in the presence of PL2-3 $(1 \mu \mathrm{g} / \mathrm{ml})$ or BWR4 (10 $\mu$ g/ml). All cells were labeled with VPD450. AM14 B cells were identified with the anti-idiotype Ab, 4-44. The percentage of divided 4-44+ cells (A) was determined by flow cytometry on day 4. (B) Representative flow cytometry plots of AM14 B cells on day 3. Cells were first gated as live, surface, and intracellular 4-44 $4^{+}$. 4-44 IgM (C) and IgG2a (E) AFCs on day 4 were determined using ELIspot assay. The concentration of 4-44+ $\operatorname{lgM}(\mathbf{D})$ and IgG2a (F) in the culture supernatant on day 4 was measured by ELISA. (C) Representative flow cytometry plots of D011.10 T cells (TCRV $\beta 6^{-} \mathrm{CFP}^{-}$) and $13 \mathrm{C} 2 \mathrm{~T}$ cells (TCRV $\beta 6^{+} \mathrm{CFPP}^{+}$) from the same culture on day 5 . Cells are first gated as live, 4-44 CD3e $\mathrm{e}^{+}$. The concentrations of IL-2 (H) and IFN- $\gamma(\mathbf{I})$ in the culture supernatant on day 4 were measured by ELISA. Data are combined from 3 or 4 independent experiments. Data are represented as the percentage of maximum for each experiment; mean $\pm \mathrm{SEM}$. Statistics were calculated with 2-way ANOVA (A and $\mathbf{C}-\mathbf{F}$ ) or 1-way ANOVA (H and I); multiple testing was corrected with Holm-Sidak's. ${ }^{*} P<0.05 ;{ }^{* *} P<0.01 ;{ }^{* * *} P<0.001 ;{ }^{* * *} P<0.0001$.

prone MRL.Fas ${ }^{\text {pr }}$ mice $(21,22) .13 \mathrm{C} 2$ and 1B9 responded to 5 and 6 of 6 different pooled sera, respectively (Supplemental Figure 1, A and B). Notably, T cells did not respond to sera from normal BALB/c mice

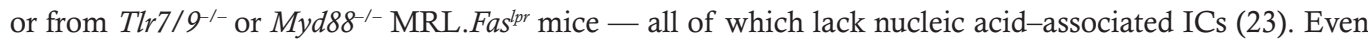
though $T \operatorname{Tr} 7 / 9^{-/-}$or $M y d 88^{-/}$MRL.Fas ${ }^{p r}$ mice lack nucleic acid-associated autoAbs, they have highly elevated levels of serum IgG2a, 5 and $3 \mathrm{mg} / \mathrm{ml}$, respectively -3.75 times and 6.25 times higher than BALB/c mice (23) - further suggesting that the $\mathrm{T}$ cells are reacting with components captured by polyclonal antinuclear Abs rather than IgG itself. These data indicate that both the autoAgs bound by PL2-3 and these T cell specificities may represent typical components of a spontaneous systemic autoimmune response.

Primary IC-specific T cells are positively selected into the CD4 compartment in vivo. In order to generate primary IC-specific T cells for functional studies, we created retroviruses (RV) encoding the rearranged TCR genes from $13 \mathrm{C} 2$ and $1 \mathrm{~B} 9$ and transduced $4 \mathrm{G} 4 \mathrm{~T}$ cells (a CD4 ${ }^{+} \mathrm{TCR}^{-}$hybridoma cell line) to test their specificity (Supplemental Figure 2, A and B). The RV-infected cells recapitulated the reactivity pattern of the original hybridomas with the autoAb panel (Supplemental Figure 2, C and D). To generate TCR 

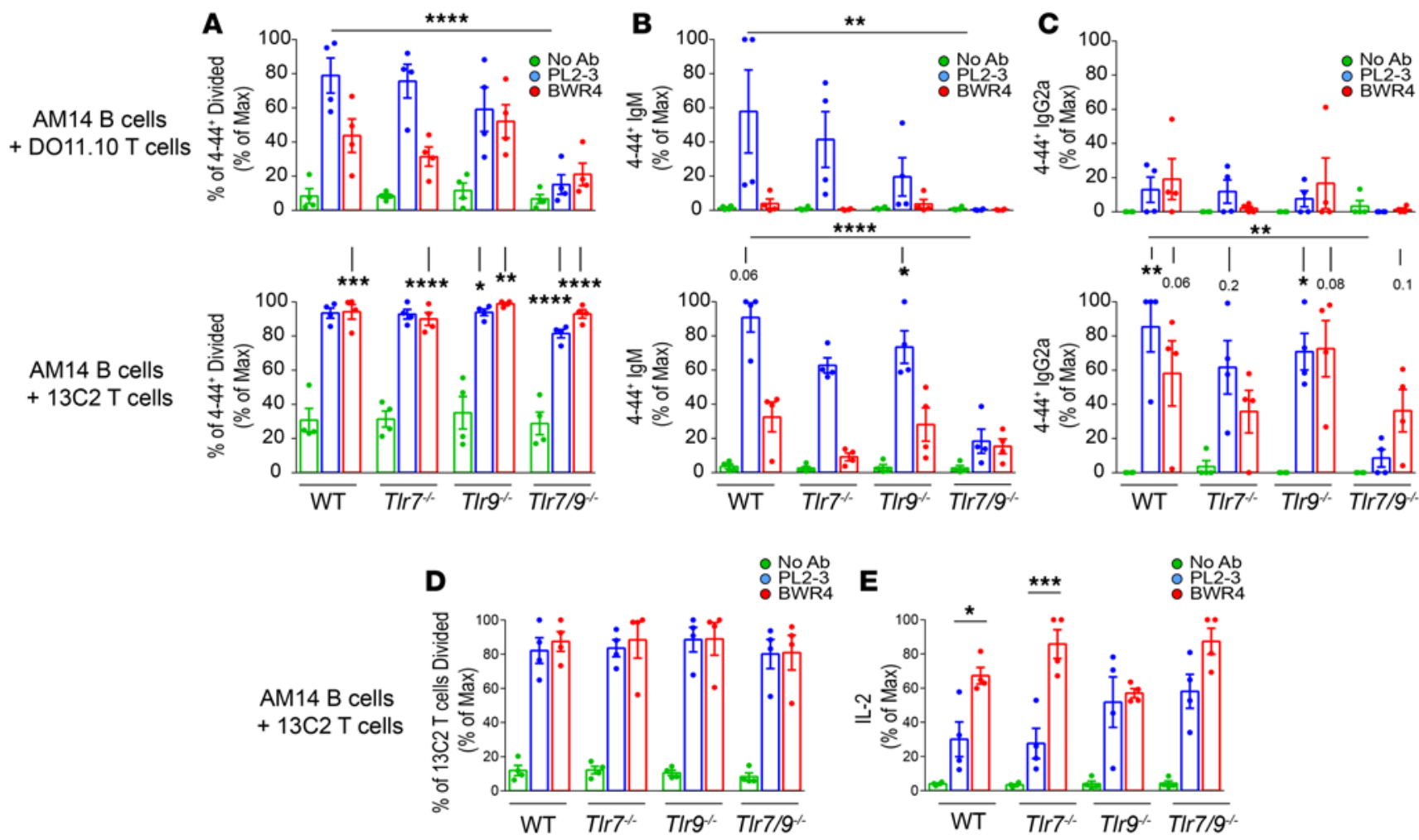

Figure 3. 13C2 T cells alleviate the need for intrinsic TLR signaling for AM14 proliferation but not Ab production. VPD450-labeled AM14 B cells (WT, TIr7-/,

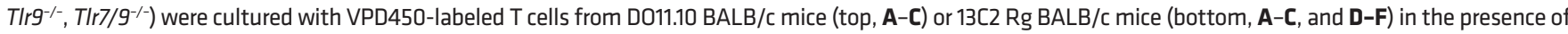
PL2-3 $(1 \mu \mathrm{g} / \mathrm{ml})$ or BWR4 $(10 \mu \mathrm{g} / \mathrm{ml})$. Analysis was performed on day 4. (A) The percentage of divided $4-44^{+}$cells was determined by flow cytometry. (B) Total $4-44^{+}$ IgM and (C) IgC2a concentration in the culture supernatant. (D) The percentage of divided 13C2 T cells was determined by flow cytometry. (E) Concentrations of IL-2 in the culture supernatant. Data are represented as the percentage of maximum for each of 4 independent experiments; mean \pm SEM. Statistics were calculated with 3-way ANOVA (A-C) or 2-way ANOVA (D-F); multiple testing was corrected with Holm-Sidak's. ${ }^{*} P<0.05$; ${ }^{* *} P<0.01 ;{ }^{* * *} P<0.001$; ${ }^{* * *} P<0.0001$.

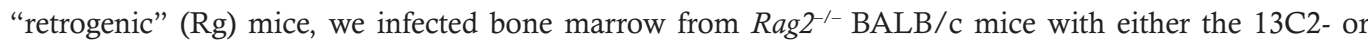
1B9-TCR RVs and then reconstituted sublethally irradiated DO11.10 $\mathrm{Tcr}^{-/-} \mathrm{BALB} / \mathrm{c}$ recipients, hereafter called $13 \mathrm{C} 2$ or $1 \mathrm{~B} 9 \mathrm{Rg}$ mice. The $\mathrm{T}$ cell repertoire of Rg mice contained $\mathrm{T}$ cells expressing $13 \mathrm{C} 2$ or $1 \mathrm{~B} 9$ TCRs as well as DO11.10 T cells that reconstituted from the host. Both $13 \mathrm{C} 2\left(\mathrm{TCRV} 6^{+} \mathrm{GFP}^{+}\right)$and $1 \mathrm{~B} 9$ cells $\left(\mathrm{TCRV} \beta 9^{+} \mathrm{GFP}^{+}\right.$) were found in the thymus and periphery (Supplemental Figure 3, A and B, and data not shown), and hence were not negatively selected. In BALB/c mice, TCRV $\beta 6^{+} \mathrm{T}$ cells and TCRV $\beta 9^{+} \mathrm{T}$ cells become CD4 or CD8 T cells at an approximate ratio of 4:1 and 1:1, respectively (Supplemental Figure 3, $\mathrm{A}$ and $\mathrm{B}$, top row). In contrast, TCRV $\beta 6^{+} \mathrm{GFP}^{+} \mathrm{T}$ cells and TCRV $\beta 9^{+} \mathrm{GFP}^{+} \mathrm{T}$ cells from the RV-infected bone marrow were almost exclusively selected into the CD4 compartment, indicating that both TCRs mediate MHC class II-restricted positive selection (Supplemental Figure 3, A and B, bottom row). Both $13 \mathrm{C} 2$ and $1 \mathrm{~B} 9 \mathrm{~T}$ cells had an increased frequency of $\mathrm{CD} 44^{+} \mathrm{CD} 62 \mathrm{~L}^{-}$cells compared with TCRV $\beta$-matched $\mathrm{T}$ cells from BALB/c mice and with the endogenous DO11.10 T cells. This population may result from retrogenesis (24) or reflect a degree of self-driven activation, though we have not observed signs of overt autoimmunity (Supplemental Figure 3, C and D). 15.9\% of $13 \mathrm{C} 2 \mathrm{~T}$ cells and $9.3 \%$ of $1 \mathrm{~B} 9 \mathrm{~T}$ cells differentiated into FoxP3 ${ }^{+} \mathrm{CD} 25^{+}$Tregs, compared with $11.2 \%$ and $14.4 \%$ of $\mathrm{V} \beta$-matched CD4 $\mathrm{T}$ cells in BALB/c mice, arguing against a strong selection into this compartment (Supplemental Figure 3, E and F). 13C2 and 1B9 $\mathrm{T}$ cells were also positively selected into the CD4 compartment on a nontransgenic BALB/c background (data not shown). However, we proceeded with DO11.10 $\mathrm{Tcr}^{-1-}$ BALB/c mice as bone marrow recipients for retrogenic mice, because they enabled us to use V-specific antibodies to distinguish the $\mathrm{T}$ cell clones of interest and prevented any complications from contaminating endogenous BALB/c T cells, which have been shown to help the AM14 B cell response (18).

Cognate interactions with $13 C 2$ T cells enhance the AM14 response to autoAb ICs in vitro. AM14 B cells proliferate in response to both PL2-3 and BWR4 in the absence of T cells $(18,25)$. To assess whether primary 
A

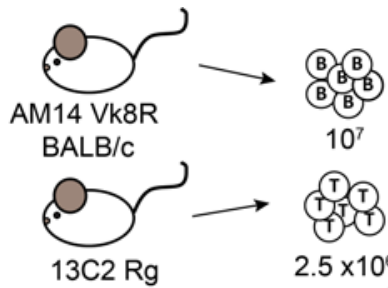

$10^{7}$

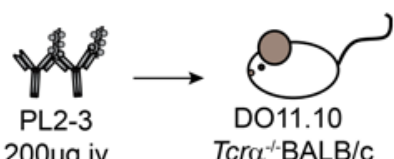

Day

\begin{tabular}{llll}
0 & 2 & 4 & 7 \\
\hline $\begin{array}{l}\text { Cell transfer } \\
\text { PL2-3 }\end{array}$ & PL2-3 & PL2-3 & Analysis
\end{tabular}

D011.10 cra $^{\prime-B A L B} / \mathrm{c} \quad 0.5 \times 10^{6}$

200ug iv $\quad \operatorname{crr}^{-}{ }^{-B A L B} / \mathrm{C}$

PL2-3

B

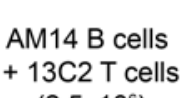

$\left(2.5 \times 10^{6}\right)$

AM14 B cells

$+\mathrm{PL} 2-3$

AM14 B cells

$+\mathrm{PL} 2-3$

$+13 \mathrm{C} 2 \mathrm{~T}$ cells

$\left(0.5 \times 10^{6}\right)$

AM14 B cells
+ PL2-3

$+13 \mathrm{C} 2 \mathrm{~T}$ cells

$\left(2.5 \times 10^{6}\right)$
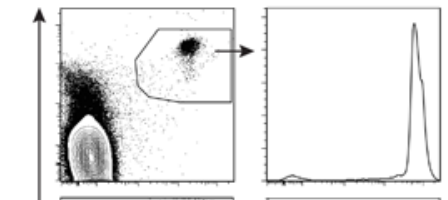

$\mathbf{C} \uparrow$
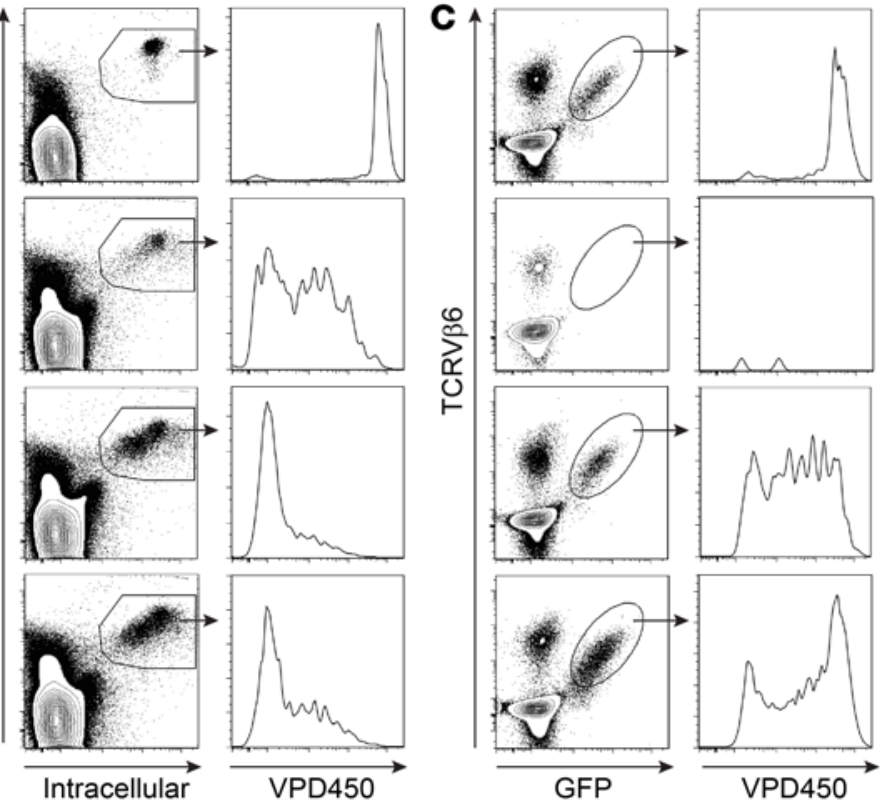

4-44

$\mathbf{F}$

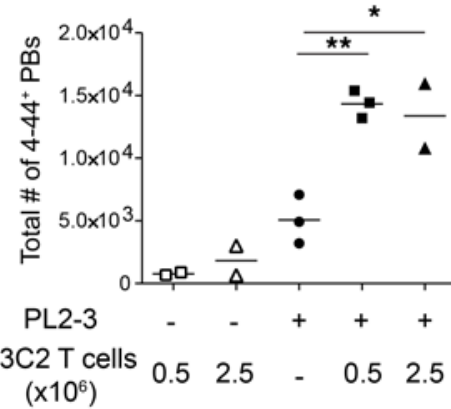

G

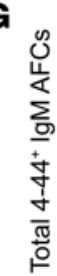

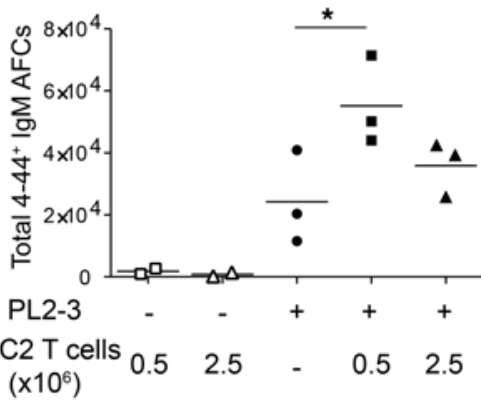

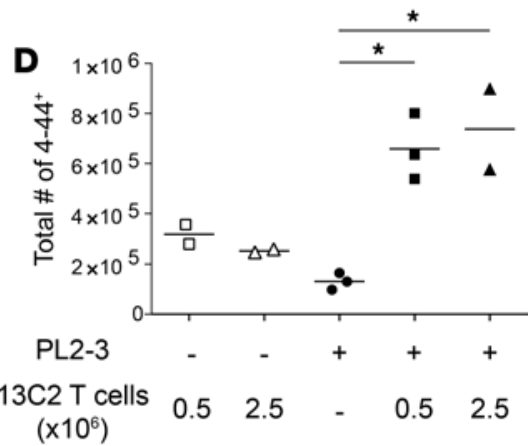

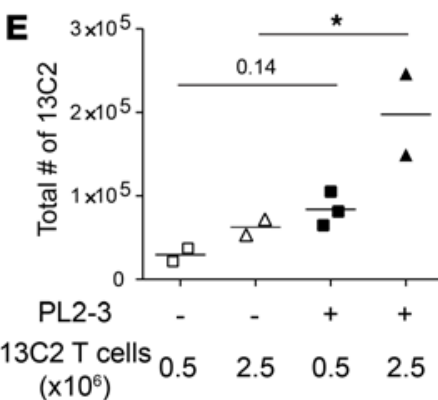

H

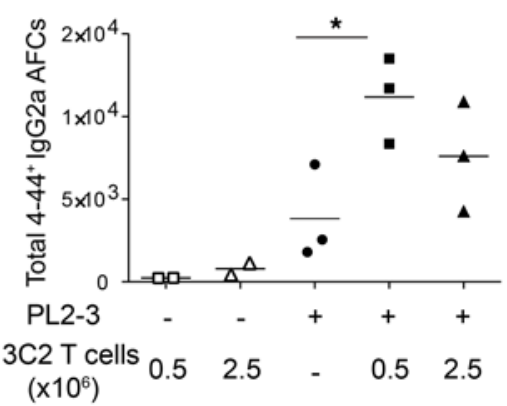

Figure 4. IC-specific T cells enhance AM14 B cell proliferation and increase AFC formation in vivo. (A) Experimental design. (B and C) Representative flow cytometry plots as indicated. Left columns, cells were first gated as live. (D and E) Total 4-44+ cells (D) and $13 C 2$ T cells (E) enumerated by flow cytometry. (F) Total 4-44+ PBs (CD138+CD44+intracellular 4-44 hi) enumerated by flow cytometry. 4-44+ IgM (C) and IgG2a (H) AFCs were determined by ELIspot. Data are from 1 experiment; bars indicate the mean, each point represents a mouse. Statistics were calculated with 1-way ANOVA; multiple testing was corrected with Holm-Sidak's. ${ }^{*} P<0.05 ;{ }^{* *} P<0.01$.

13C2 T cells would influence this AM14 response, we cultured AM14 B cells with PL2-3 or BWR4, either alone (data not shown) or in the presence of T cells from 13C2 Rg or control DO11.10 mice. 13C2 T cells significantly enhanced B cell proliferation and accumulation in response to BWR4 but not PL2-3 (Figure 2, A and B). Cultures containing 13C2 T cells and BWR4 also had an increase in IgM Ab-forming cells (AFCs) and total IgM (Figure 2, C and D). Although 13C2 $\mathrm{T}$ cells did not affect the number IgM AFCs produced by PL2-3 stimulation, the total IgM in the supernatant was increased (Figure 2, C and D). 13C2 $\mathrm{T}$ cells promoted a substantial increase in IgG2a isotype-switched AFCs and total IgG2a for both PL2-3 and BWR4 stimulation cultures (Figure 2, E and F). Collectively, these data indicate that, although AM14 $\mathrm{B}$ cells can differentiate into IgM AFCs without $\mathrm{T}$ cell help, the presence of $13 \mathrm{C} 2 \mathrm{~T}$ cells enhanced $\mathrm{Ab}$ production and strongly promoted isotype switch, especially in response to anti-RNA ICs.

We also examined the $\mathrm{T}$ cell responses. Strikingly, $13 \mathrm{C} 2 \mathrm{~T}$ cells but not DO11.10 $\mathrm{T}$ cells proliferated and 
A

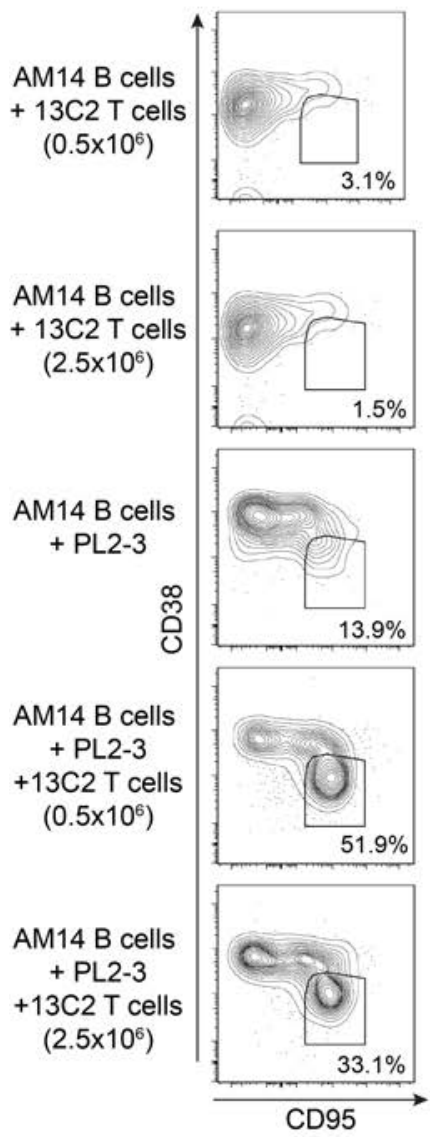

B

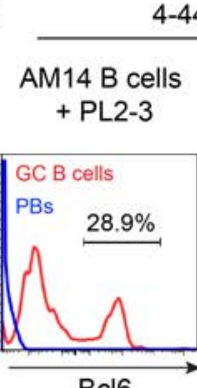

Bcl6
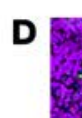

$4-44^{+}$cells

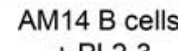

$+\mathrm{PL} 2-3$

$+13 \mathrm{C} 2 \mathrm{~T}$ cells

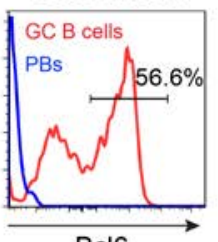

Bcl6

C

TCRV $\beta 6^{+} \mathrm{GFP}^{+}$cells AM14 B cells
$+13 \mathrm{C} 2 \mathrm{~T}$ cells AM14 B cells

$+\mathrm{PL} 2-3$

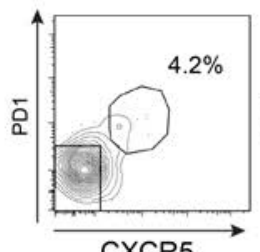

CXCR5

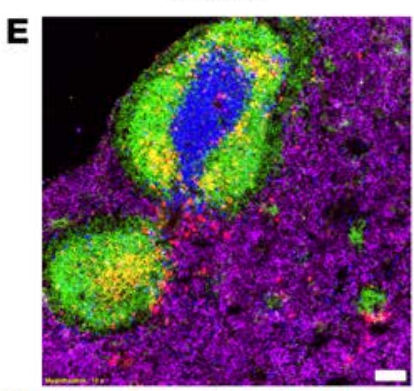

$+13 \mathrm{C} 2 \mathrm{~T}$ cells

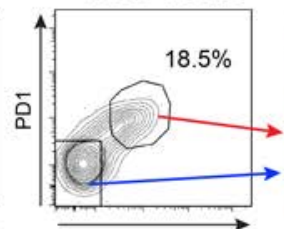

CXCR5

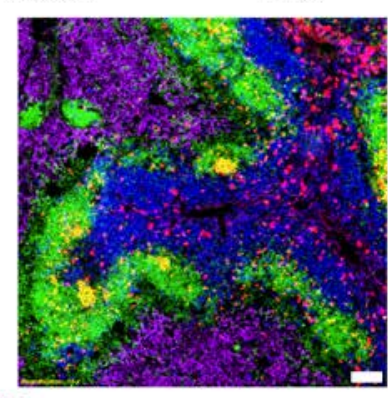

$\mathbf{F}$

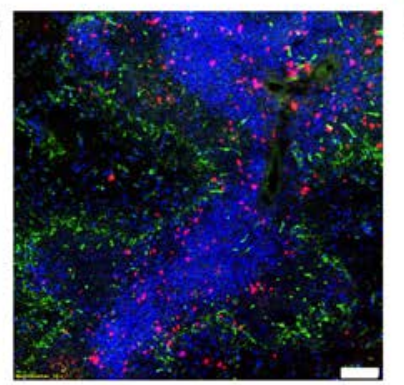

CD45R TCR $\beta \quad$ 4-44 (AM14) F4/80

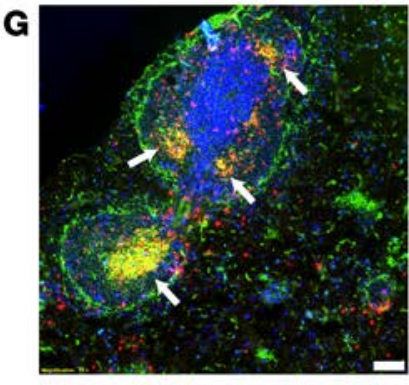

PNA TCR $\beta$ 4-44 (AM14)

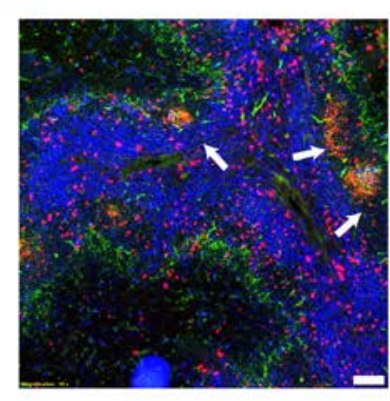$$
\text { . }
$$

Figure 5. 13C2 T cells can adopt a Tfh cell phenotype and induce AM14 B cells to differentiate into germinal center cells. (A) Representative flow cytometry plots from the experiment described in Figure 4; percentages reflect the mean. Cells were first gated as live and 4-44 ${ }^{+}$. (B and C) Experimental design is as depicted in Figure 4A, except $5 \times 10^{6} \mathrm{AM} 14 \mathrm{~B}$ cells and $2.5 \times 10^{5} \mathrm{~T}$ cells were transferred. (B) Representative flow cytometry histograms of PBs

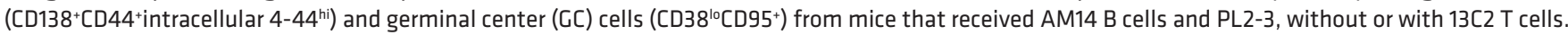
Percentages reflect means from 4 experiments, with 14 and 16 total mice per group, respectively. (C) Representative flow cytometry plots and histograms gated on Rg T cells from mice that received AM14 B cells and 13C2 T cells, without and with PL2-3. Percentages reflect means from 6 experiments, with 8 and 25 total mice per group, respectively. All cells were first gated as live. (D-G) Immunofluorescent histology from the experiment described in Figure 4; representative of 2 similar experiments. ( $\mathbf{D}$ and $\mathbf{F}$ ) Representative images from mice that received AM14 B cells and PL2-3. (E and $\mathbf{G}$ ) Representative images from mice that received AM14 B cells, 13 C2 T cells, and PL2-3. Each column of images is from a different mouse. White arrows indicate 4-44+ PNA ${ }^{+}$ GC cells. Original magnification, $\times 20$. Scale bar: $100 \mu \mathrm{m}$.

produced IL-2 and IFN- $\gamma$ when cultured with AM14 B cells and PL2-3 or BWR4 (Figure 2, G-I). Hence, cognate interactions with AM14 B cells produced a vigorous response to self-derived antinuclear ICs.

$13 C 2 T$ cells bypass the need for intrinsic TLR signaling for AM14 proliferation but not differentiation in vitro. The AM14 response to autoAb ICs is dependent on TLR7/9 $(11,20,25,26)$. We hypothesized that IC-specific T cells could substitute for TLR signals. To test this, we stimulated AM14 B cells that were deficient in $T / r 7, T / r 9$, or both in the presence of DO11.10 T cells or $13 \mathrm{C} 2 \mathrm{~T}$ cells. While proliferation was almost absent in the T/r7/9-1- $\mathrm{B}$ cells cultured with DO11.10 $\mathrm{T}$ cells (Figure 3A, upper), the presence of $13 \mathrm{C} 2 \mathrm{~T}$ cells completely alleviated the need for TLR signaling for B cell proliferation (Figure 3A, lower). However, IC-specific T cells did not restore Ab production to the level of that of TLR-intact B cells. Nonetheless, the presence of IC-specific T cells signaled an increasing trend compared with control cultures with DO11.10 T cells (Figure 3, B and C, and data not shown). Surprisingly, T cell proliferation and IL-2 production were unaffected by the TLR genotype of the B cell, indicating that TLR activation in the B cell is not necessary for Ag presentation (Figure 3, D and E).

IC-specific T cells amplify the AM14 response and alter B cell fate decisions in vivo. To study the effect of 13C2 $\mathrm{T}$ cells on the AM14 B cell response in vivo, we transferred AM14 B cells and 13C2 T cells into DO11.10 
A

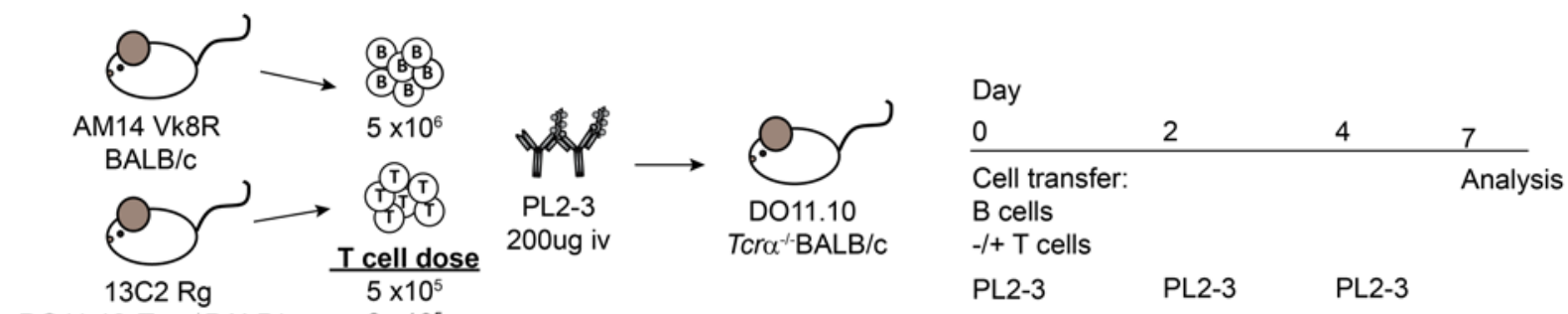
D011.10 Tcro $^{-/ B A L B} / \mathrm{c} \quad 2 \times 10^{5}$ $0.5 \times 10^{5}$

B

RF B cells +13 C 2 T cells + PL2-3
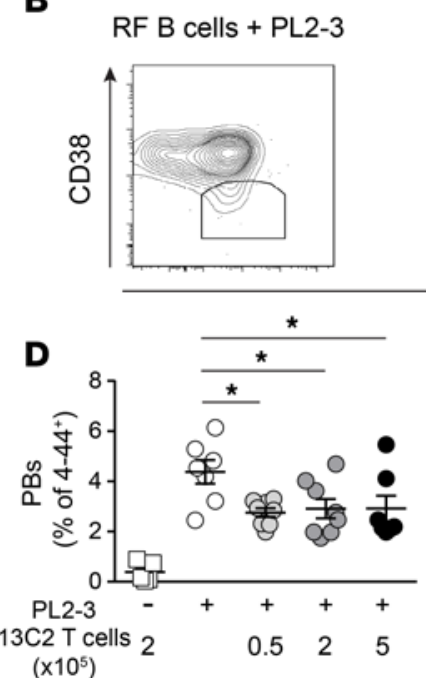

$\left(\mathrm{x} 10^{5}\right)$
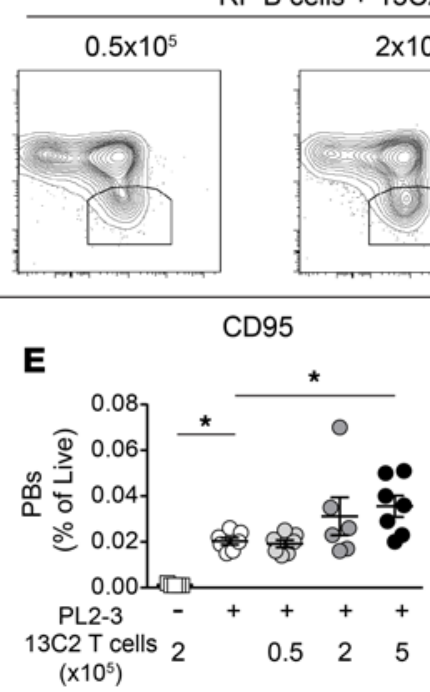

$2 \times 10^{5}$

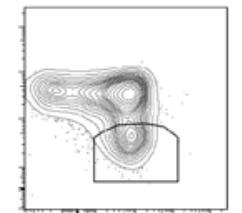

$\mathbf{F}$

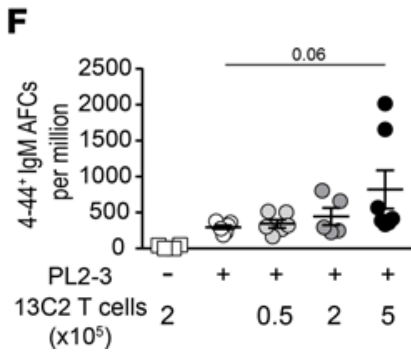

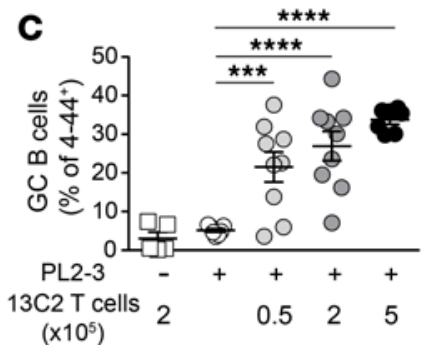

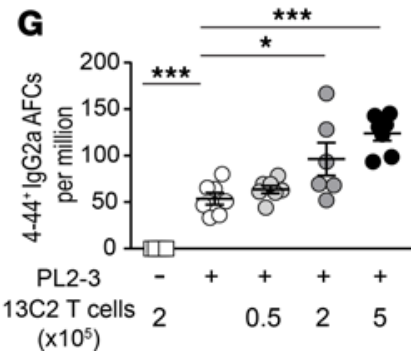

Figure 6. The effects of cognate T cell help for AM14 B cells depend on a threshold number of T cells and on T cell clone identity. (A) Experimental design. (B) Representative flow cytometry plots. Cells were first gated as live and $4-44^{+}$. 4-44+ germinal center (GC) cells (B and C) and PBs (D and E) enumerated by flow cytometry. 4-44+ IgM (F) and (G) IgG2a AFCs determined by ELIspot. Data are presented as mean \pm SEM from 2 independent experiments; each point represents a mouse, with 5-8 total mice per group. Statistics were calculated with 1-way ANOVA; multiple testing was corrected with Holm-Sidak's. ${ }^{*} P<0.05 ;{ }^{* *} P<0.001 ;{ }^{* * * *} P<0.0001$.

$\mathrm{Tcr \alpha}^{-1-} \mathrm{BALB} / \mathrm{c}$ recipients (Figure 4A). With PL2-3 treatment, AM14 B cells that were cotransferred with $13 \mathrm{C} 2 \mathrm{~T}$ cells divided to a greater extent and yielded more total cells; there was no division in the absence of PL2-3 (Figure 4, B and D). 13C2 T cells also only proliferated with PL2-3 treatment; mice that received the higher dose of T cells had significantly more total 13C2 T cells after PL2-3 treatment (Figure 4, C and E). In addition, transfer of $13 \mathrm{C} 2 \mathrm{~T}$ cells increased the magnitude of the plasmablast $(\mathrm{PB})$ response, and the lower dose of T cells resulted in more IgM and IgG2a AFCs compared with AM14 B cells alone (Figure 4, F-H).

AM14 B cells preferentially differentiate into PBs rather than germinal center (GC) B cells both in the spontaneous response in lupus-prone MRL.Fas ${ }^{j p r}$ mice and when induced by PL2-3 in vivo (10, 25, 27, 28). The reason for this has been unclear but could include the dual BCR/TLR signals provided by the IC or a lack of optimal T cell help to drive GC B cell differentiation. We found that the percentage of AM14 cells with a $\mathrm{CD} 38^{1 \circ} \mathrm{CD} 95^{+} \mathrm{Bc} 16^{\text {hi }} \mathrm{GC}$ phenotype was substantially enhanced in the presence of cognate IC-specific $\mathrm{T}$ cells (Figure 5, A and B). Commensurate with the differentiation of AM14 B cells into GC cells, a proportion of the $13 \mathrm{C} 2 \mathrm{~T}$ cells differentiated into follicular helper $\mathrm{T}$ cells (Tfh cells, PD- ${ }^{\text {hi }} \mathrm{CXCR} 5^{+} \mathrm{Bc} 16^{+}$) (Figure $5 \mathrm{C}$ ). Regardless of $\mathrm{T}$ cell transfer, 4-44-bright $\mathrm{PBs}$ could be found in the $\mathrm{T}$ cell zone, clustered around the periarteriolar lymphoid sheath and on the border of the follicle and red pulp (Figure 5, D and E). However, 4-44 ${ }^{+} \mathrm{PA}^{+}$ GC cells were only detected when 13C2 T cells were cotransferred (Figure 5, F and G). Thus, 13C2 T cells alter AM14 B cell fate, converting a PB-only response into one that contains both PBs and GC cells.

The increased PB response and GC differentiation in the presence of IC-specific T cells could be the result of a number of factors, including increased frequency of cognate $\mathrm{T}$ cells, altered kinetics of $\mathrm{T}$ cell help availability, or the quality of the $\mathrm{T}$ cell help. To test whether the quantity of $13 \mathrm{C} 2 \mathrm{~T}$ cells altered the AM14 B cell response, we titrated the number of $13 \mathrm{C} 2 \mathrm{~T}$ cells transferred (Figure $6 \mathrm{~A}$ ). $5 \times 10^{5}$ to $0.5 \times 10^{5}$ transferred $13 \mathrm{C} 2 \mathrm{~T}$ cells induced a robust GC phenotype at day 7 (Figure 6, B and C). We did an additional 
A

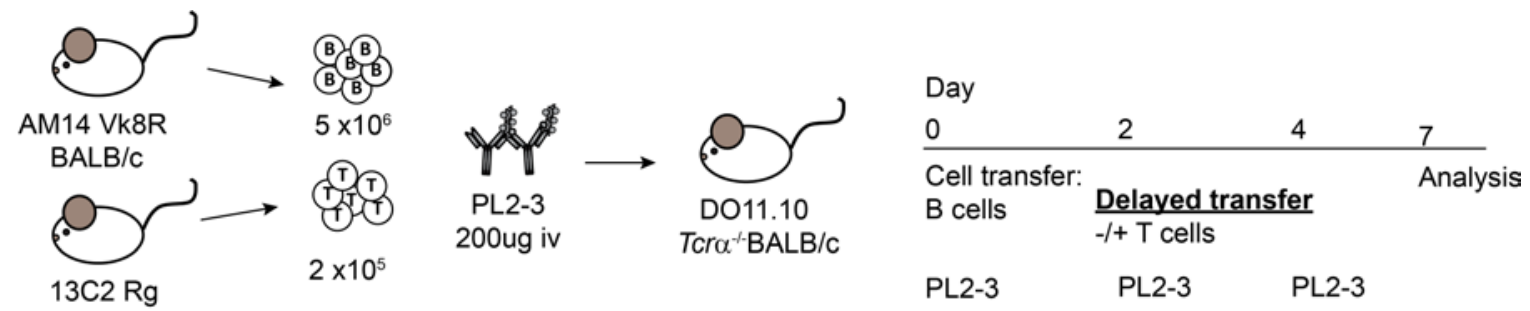

D011.10 Tcr $^{-1-B A L B / C}$

B

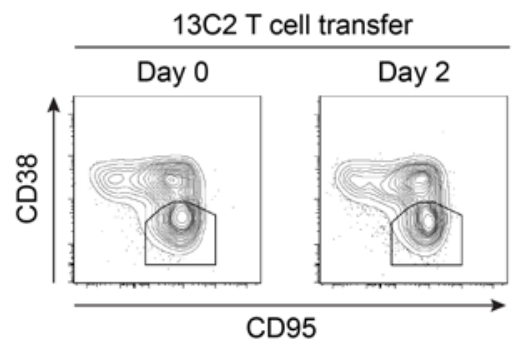

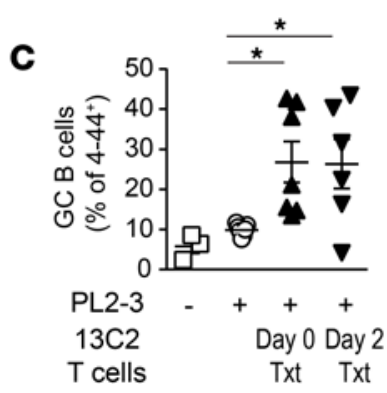

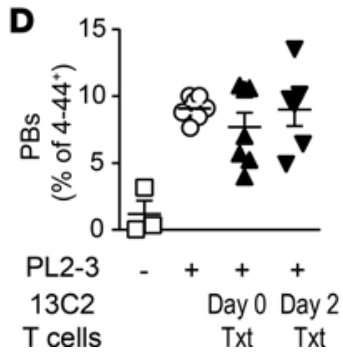

Figure 7. AM14 GC B cell development is not dependent on early T cell help. (A) Experimental design. (B) Representative flow cytometry plots. Cells were first gated as live and $4-44^{+} .4-44^{+}$germinal center (GC) cells (C) and PBs (D) enumerated by flow cytometry. Data are presented as mean \pm SEM from 2 independent experiments; each point represents a mouse, with 3-7 total mice per group. Statistics were calculated with 1-way ANOVA; multiple testing was corrected with Holm-Sidak's. ${ }^{*} P<0.05$.

experiment transferring $0.2 \times 10^{5} 13 \mathrm{C} 2 \mathrm{~T}$ cells per mouse, which did not result in a AM14 GC population, indicating this was below the threshold needed to alter B cell differentiation (data not shown). 13C2 T cells had a slightly suppressive effect on PB differentiation, as measured by the portion of $4-44^{+}$cells; however, the total PB population, measured as percentage of live cells, was increased with the highest dose of $\mathrm{T}$ cells (Figure 6, D and E). Transfer of $2 \times 10^{5}$ or $5 \times 10^{5} 13 \mathrm{C} 2 \mathrm{~T}$ cells enhanced isotype switch to IgG2a AFCs but did not reach statistical significance with IgM (Figure 6, F and G).

To investigate whether early availability of T cell help promoted GC differentiation, we delayed the transfer of $13 \mathrm{C} 2 \mathrm{~T}$ cells for 2 days (Figure 7A). However, 13C2 $\mathrm{T}$ cells were equally capable of inducing GC differentiation if they were cotransferred with AM14 B cells on day 0 or transferred 2 days later (Figure 7, $\mathrm{B}$ and $\mathrm{C}$ ). $13 \mathrm{C} 2 \mathrm{~T}$ cells given on either day did not enhance the proclivity to differentiate into PBs (Figure 7D). Taken together, these results show that $13 \mathrm{C} 2 \mathrm{~T}$ cells induce AM14 B cells stimulated with PL2-3 to differentiate into GC cells, increase the magnitude of the $\mathrm{PB}$ response, and promote isotype switching.

To investigate whether the capability to skew cognate B cell responses toward GC differentiation was unique to the $13 \mathrm{C} 2$ clone, we analyzed the effect of an additional IC-specific T cell clone, 1B9 (Figure $8 \mathrm{~A}$ ). The transfer of $1 \mathrm{~B} 9$ or $13 \mathrm{C} 2 \mathrm{~T}$ cells resulted in an equivalent proportion of GC AM14 B cells (Figure $8, \mathrm{~B}$ and C). Strikingly, the cotransfer of $1 \mathrm{~B} 9 \mathrm{~T}$ cells resulted in a substantial increase in PB differentiation compared with AM14 B cells transferred alone or cotransferred with 13C2 T cells (Figure 8D). Cotransfer of the 1B9 T cells resulted in 7.5-fold more AM14 B cells compared with AM14 B cells transferred alone and 2.25-fold more compared with $13 \mathrm{C} 2 \mathrm{~T}$ cells; this resulted in both substantially more GC $\mathrm{B}$ cells and $\mathrm{PBs}$ (Figure 8, E-G). 1B9 T cells increased IgM AFCs by approximately 7-fold compared with AM14 B cells alone and by almost 5 -fold compared with 13C2 T cells (Figure 8H). Finally, 1B9 T cells resulted in 100 times more IgG2a AFCs compared with AM14 B cells transferred alone and almost 40 times more compared with 13C2 T cells (Figure 8I).

The differential effects of the two clones on the AM14 B cell response could be due to a number of factors, including the quality and quantity of activated $\mathrm{T}$ cells generated by each clone. Indeed, only $13 \mathrm{C} 2$, and not 1B9, required the cotransfer of AM14 B cells in order to proliferate in response to PL2-3 (Figure 9, $\mathrm{A}$ and B). 1B9 $\mathrm{T}$ cells proliferated more than 13C2 $\mathrm{T}$ cells, as shown by increased VPD450 dilution, generating 10 times more IC-specific T cells (Figure 9, A and B). Qualitatively, however, both $1 \mathrm{~B} 9$ and $13 \mathrm{C} 2 \mathrm{~T}$ cells differentiated into an equivalent frequency of Tfh cells (Figure 9, C and D). These data suggest that the antigenic peptide for $1 \mathrm{~B} 9$ can be presented in vivo by endogenous APCs that take up ICs, but the specific peptide recognized by $13 \mathrm{C} 2$ may be uniquely created or concentrated 
A

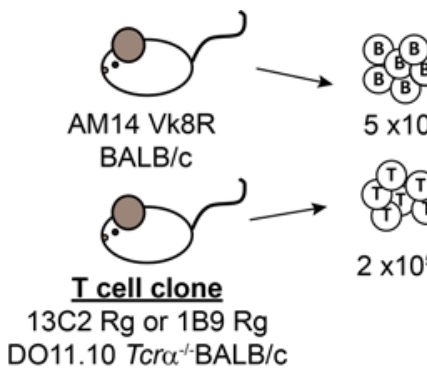

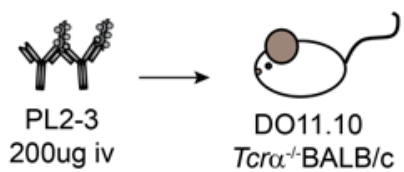

Day

\begin{tabular}{|c|c|c|c|}
\hline 0 & 2 & 4 & \\
\hline Cell transfer: & & & Analys \\
\hline
\end{tabular}

B

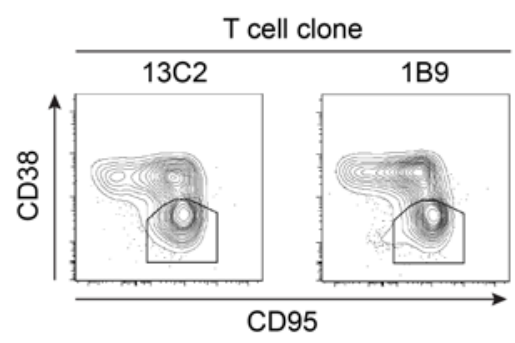

$\mathbf{E}$
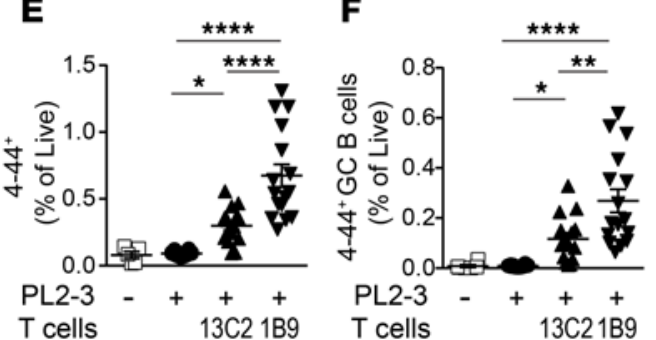
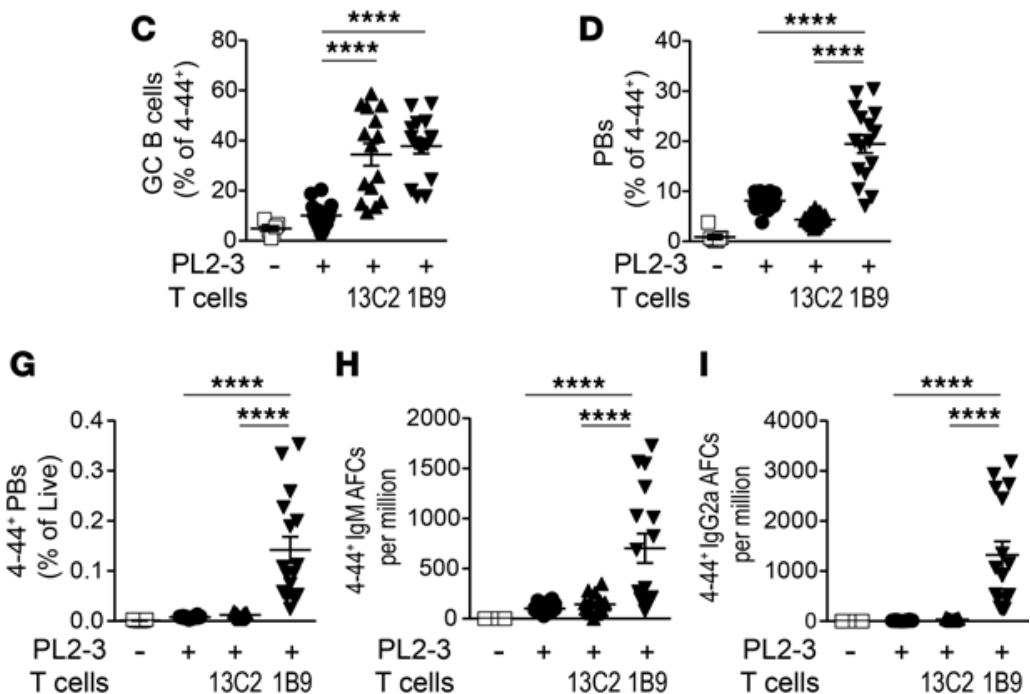

Figure 8. T cell clone 1B9 dramatically alters AM14 B cell accumulation and differentiation. (A) Experimental design. (B) Representative flow cytometry plots as indicated. Cells were first gated as live and $4-44^{+} .4-44^{+}$germinal center (GC) cells (C and F), PBs (D and G), and total 4-44+ cells enumerated by flow cytometry. 4-44+ IgM (H) and (I) IgG2a AFCs were determined by ELIspot. Data are presented as mean \pm SEM from 4 independent experiments; each point represents a mouse, with 6-16 total mice per group. Statistics were calculated with 1-way ANOVA; multiple testing was corrected with Holm- Sidak's. ${ }^{*} P<0.05 ;{ }^{* *} P<0.01 ;{ }^{* * *} P<0.0001$.

by AM14 B cell antigen processing.

$I C$-specific $T$ cells partially bypass the requirement for intrinsic TLR signaling for the AM14 response in vivo. To test whether IC-specific T cells could rescue TLR-deficient AM14 B cell responses in vivo, we transferred AM14 B cells that were WT, $T \operatorname{tr} 7^{-/}, \operatorname{Tl} r^{-/-}$, or $\operatorname{Ttr} 7 / 9^{-/-}$alone or with $13 \mathrm{C} 2$ or $1 \mathrm{~B} 9 \mathrm{~T}$ cells, with or without PL2-3 (Supplemental Figure 4). In the absence of T cells, Tlr7/9-1- AM14 B cells almost completely disappeared. In the presence of $13 \mathrm{C} 2 \mathrm{~T}$ cells, the frequency of $T / 77 / 9^{-1-} \mathrm{AM} 14 \mathrm{~B}$ cells increased 7 -fold; in the presence of 1B9 $\mathrm{T}$ cells, the frequency increased 30-fold (Figure 10, A and B). There was little effect of single TLR deficiency, except that, in the presence of T cells, TLR9-deficient AM14 B cells actually yielded an even greater number of cells than did TLR-intact AM1 B cells.

The loss of both TLR7 and TLR9 or TLR7 alone resulted in significant decreases in the percentage of AM14 B cells with GC phenotype in the presence of either T cell clone (Figure 10C). Nonetheless, $\mathrm{T}$ cells could partly rescue TLR deficiency, since, even in the absence of both TLRs, transfer of $13 \mathrm{C} 2$ or 1B9 cells resulted in more AM14 GC B cells than were produced without $\mathrm{T}$ cells. These results indicate that AM14 differentiation into GC cells in the context of sufficient T cell help is partially dependent on intrinsic TLR7, but not TLR9, signaling.

For AM14 B cells transferred alone, either TLR was sufficient to support PB differentiation (Figure 10D); in contrast, the few Tlr7/9-1- AM14 B cells that survived did not become PBs. In mice that received $13 \mathrm{C} 2 \mathrm{~T}$ cells and Tr7/19-- AM14 B cells, PB differentiation was also impaired, but this did not reach statistical significance. The transfer of $13 \mathrm{C} 2 \mathrm{~T}$ cells with $T l r^{-/-} \mathrm{AM} 14 \mathrm{~B}$ cells led to an increasing trend in PBs - the opposite effect from that observed on GC differentiation. Strikingly, 1B9 T cells completely bypassed the need for TLR signaling for PB differentiation. While 1B9 T cells enabled Tir7/9-- AM14 B cells to differentiate into phenotypic PBs, these B cells were impaired in their ability to become IgM AFCs and lost the ability make IgG2a 
A RF B cells
+13 C 2 T cells

$13 \mathrm{C} 2 \mathrm{~T}$ cells $+\mathrm{PL} 2-3$

RF B cells

$+13 \mathrm{C} 2 \mathrm{~T}$ cells

$+\mathrm{PL} 2-3$

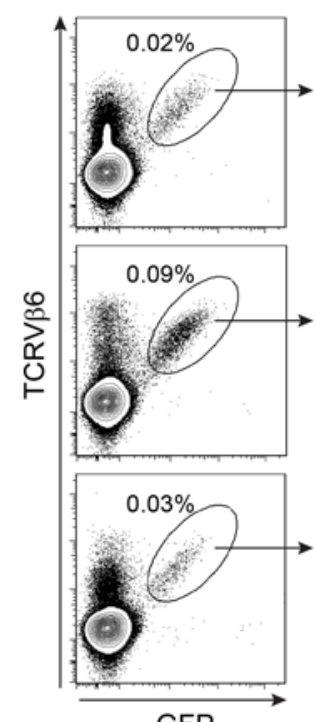

GFP
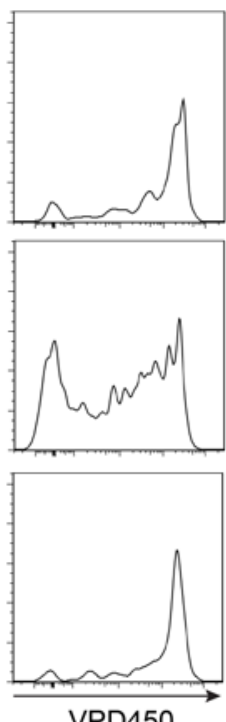

VPD450
C $\mathrm{TCRV} 9^{+} \mathrm{GFP}^{+}$cells
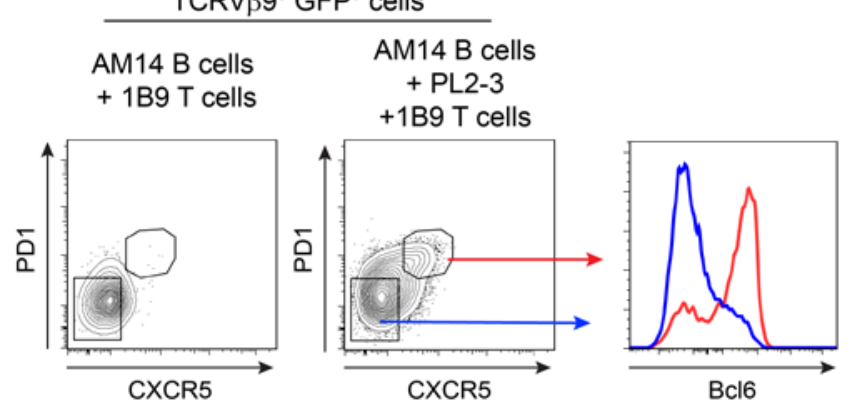

B
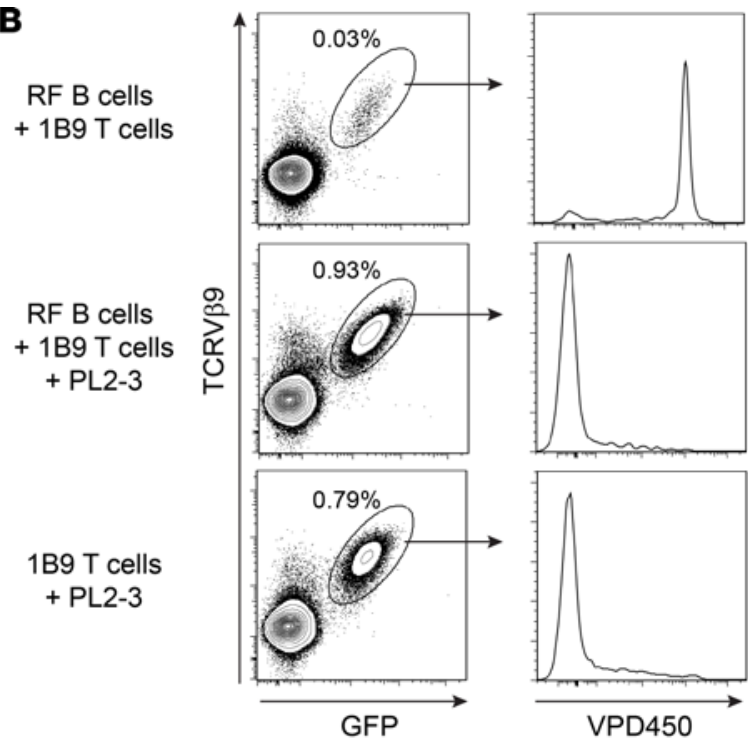

\section{D}

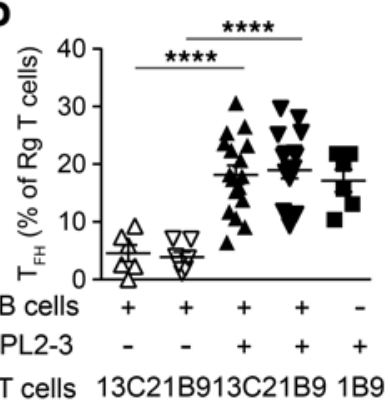

Figure 9. 13C2, but not 1B9, T cells require AM14 B cells for activation with PL2-3 in vivo. Experimental design was the same as described in Figure 6A using both T cell clones, $13 \mathrm{C2}$ and 1B9. (A and B) Representative flow cytometry plots and histograms as indicated; percentages reflect the mean from 4 experiments. Cells were first gated as live. (C) Representative flow cytometry plots and histograms as indicated from 4 experiments. Cells were first gated as live and $C D 4^{+}$. (D) $13 C 2$ and $1 B 9$ Tfh cells $\left(P D 1^{+} C X C R 5^{+}\right)$enumerated from flow cytometry. Data are shown as mean \pm SEM from 4 experiments, with 6-18 total mice per group. Statistics were calculated with 1-way ANOVA; multiple testing was corrected with Holm-Sidak's. ${ }^{* * *} P<0.0001$.

AFCs (Figure 10, E and F). Collectively, these results demonstrate that cognate IC-specific T cells can partially overcome the need for TLR signaling in response to PL2-3 in vivo, particularly with respect to GC differentiation and, in the case of 1B9, PB differentiation. However, even in the presence of T cell cognate help, TLR stimulation significantly contributes to expansion and complete differentiation in response to a native autoAg.

On the other hand, consistent with the in vitro data, the TLR genotype of the AM14 B cells did not affect the total number of IC-specific T cells (Figure 10G). Thus, although there were fewer Tlr7/9-1-AM14 $\mathrm{B}$ cells by day 7 to present $\mathrm{Ag}$ to $\mathrm{T}$ cells, the initial population must have been sufficient to initiate $\mathrm{T}$ cell activation and proliferation. Lack of TLR7 and TLR9 on AM14 B cells did, however, reduce the differentiation of $13 \mathrm{C} 2$ but not 1B9 T cells into Tfh cells (Figure 10H).

\section{Discussion}

Although autoreactive $\mathrm{T}$ cells play a key role in the pathogenesis of systemic autoimmunity, relatively little is known about their origin, specificity, phenotype, fate, and function. Here, we have used what we believe to be a novel method to identify relevant autoreactive $\mathrm{T}$ cells that help autoAb-producing $\mathrm{B}$ cells. Our results directly confirm that autoreactive helper $\mathrm{T}$ cells exist in the periphery of normal mice and extend this notion by showing how they can function. Upon encounter with Ag in the appropriate form, they can be activated to break functional tolerance (or clonal "ignorance"), in a process that does not require any non-self adjuvant. Both $\mathrm{T}$ cell clones significantly enhanced the magnitude and altered the quality of the AM14 response in vivo. Perhaps most critically for our understanding 
A

No PL2-3

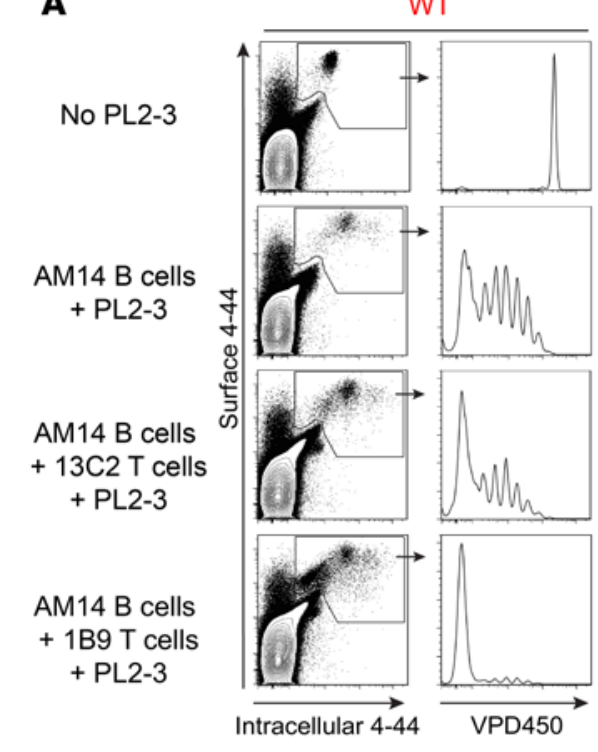

WT

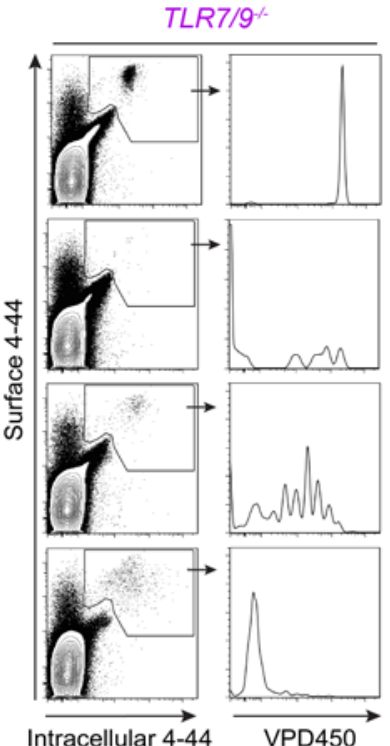

\section{E \\ E}

C

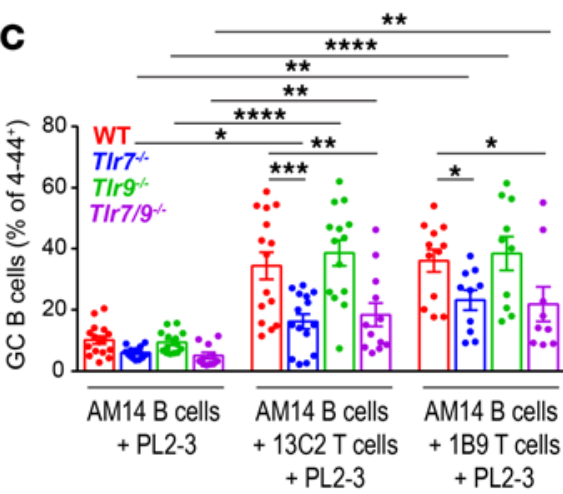

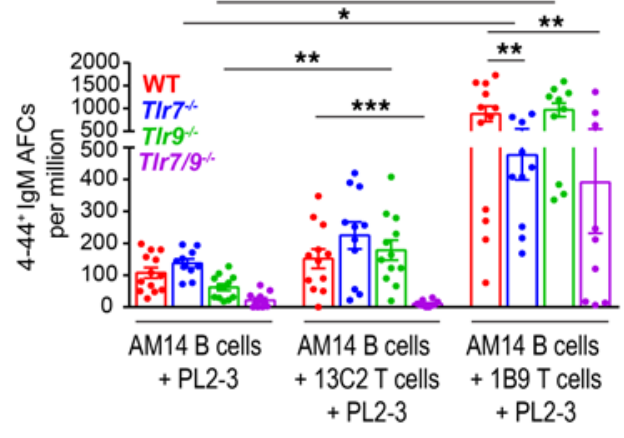

F
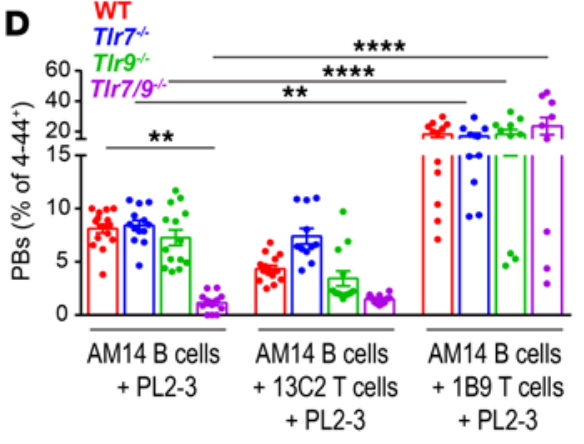

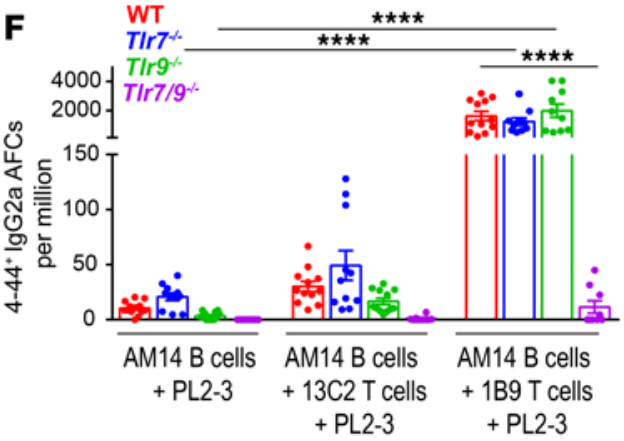

B

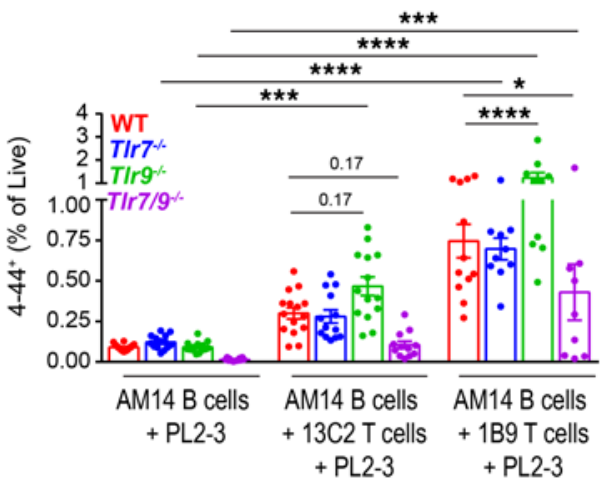

G
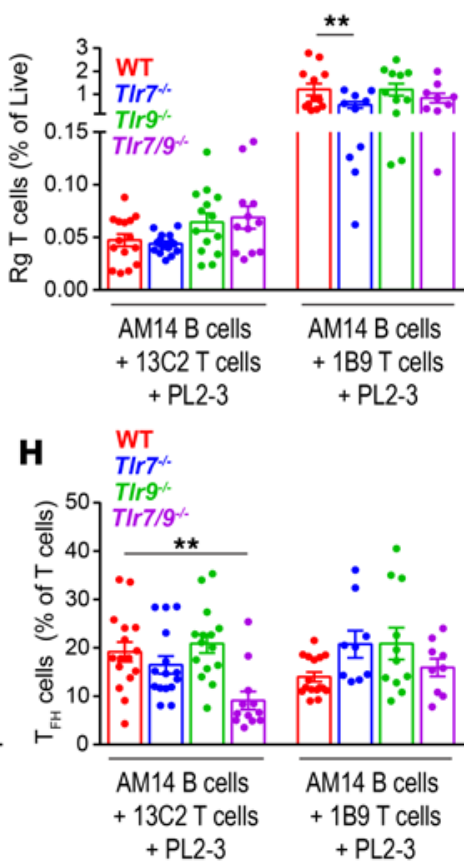

Figure 10. IC-specific T cells partially bypass the requirement for intrinsic TLR signaling in the AM14 in vivo response. (A) Representative flow cytometry plots and histograms from mice that received WT or T/r7/9-/- AM14 B cells. In the left columns for each genotype, cells were first gated as live. (B) Total 4-44+ cells enumerated from flow cytometry. 4-44+ germinal center (GC) cells (C) and PBs (D) enumerated by flow cytometry. 4-44+ IgM (E) and (F) IgG2a AFCs were determined by ELIspot. (C) Total $13 \mathrm{C2}$ and 1B9 T cells enumerated by flow cytometry. Cells were first gated as live and CD4 $4^{+}$. (H) $13 \mathrm{C2}$ and 1B9 Tfh cells (PD1+CXCR5 ${ }^{+}$) enumerated from flow cytometry. Data are presented as mean \pm SEM from 4 independent experiments, with 7-16 total mice per group; each point represents a mouse. Statistics were calculated with 2-way ANOVA; multiple testing was corrected with Holm-Sidak's. ${ }^{*} P<0.05 ;{ }^{* *} P<0.01 ;{ }^{* * *} P<0.001 ;{ }^{* * * *} P<0.0001$.

of systemic autoimmunity pathogenesis, we found that IC-specific T cells could rescue many aspects of the TLR-deficient AM14 B cell response in vivo. Thus, the requirement for intrinsic B cell TLR signaling may depend on the stage and type of immune response and may be overridden, in substantial part, by sufficient cognate $\mathrm{T}$ cell help. This has implications for the efficacy of TLR-targeted therapy in interrupting ongoing disease.

Our studies complement other examples - mostly derived from models of organ-specific autoimmunity - of self-reactive $\mathrm{T}$ cells that escape central deletion, exist in a normal repertoire, and can become activated. 
Such cells can exist for multiple reasons: peptides that bind MHC weakly cannot mediate deletion in the thymus but can induce activation in the periphery, presumably through increased local concentration, either naturally or through immunization (29-31). Incomplete thymic expression of protein splice variants can also result in thymic escape $(32,33)$. How autoreactive $\mathrm{T}$ cells escape tolerance and are activated in lupus has been investigated but is not well understood. Immunization with native snRNPs, a target of lupus autoAbs, did not elicit a response from normal mice; however, administration of specific snRNP peptides led to $\mathrm{T}$ cell activation and $\mathrm{Ab}$ production — indicating that some self-peptides are not naturally processed and presented (4). Notably, when Ag-specific B cells were present, T cell responses from normal mice to murine snRNPs were detectable - further demonstrating that normal mice harbor autoreactive $\mathrm{T}$ cells and suggesting a special role for B cell presentation in the activation of such "ignorant" autoreactive T cells (34).

Our $\mathrm{T}$ cell clones provide greater insight into the ontogeny of lupus-related autoreactivity. Both clonotypes were positively selected and appeared mainly quiescent in the periphery at steady state. However, they differed in terms of their activation requirements and function in the periphery. Since 13C2 T cells required AM14 B cells for activation in vivo, their specific epitope may be the result of unique processing by these Ag-specific B cells (35). In contrast, 1B9 T cells did not require the cotransfer of AM14 B cells for their activation, indicating that their epitope can be presented by endogenous APCs. Since 1B9 T cells were not spontaneously activated in BALB/c mice in the absence of ICs, at steady state their endogenous Ag must not be presented at a sufficient concentration and/or in immunogenic context. It is also possible that the stimulatory epitope may result from altered peptide processing of the parent protein due to its inclusion in ICs.

The exogenous addition of antichromatin, similar to the accumulation of serum IC over the course of lupus, may be necessary to overcome barriers to $\mathrm{T}$ cell activation. Autoreactive $\mathrm{T}$ cells that are activated later in disease progression could fall into this category (i.e., they can be stimulated by high titers of serum self-ICs); they would be activated by any FcR-bearing APC, perhaps like 1B9 but not 13C2. In this regard, it is interesting that we saw that autoreactive T cells that help autoreactive B cells are diverse in function and activation requirements, even though identical conditions were used to isolate them. Despite these differences, it is important to note that many aspects of these two different clones were similar, revealing general themes concerning the interplay of T cells, B cells, and TLR signals in systemic autoimmune responses.

Of greatest mechanistic and therapeutic significance, we found that AM14 B cells could induce T cell activation completely independently of TLRs. This was unexpected, since B cell-specific MyD88 deletion in MRL.Fas ${ }^{p r}$ mice resulted in a significant decrease in T cell activation (36). To reconcile this, we suggest that TLR-driven activation of autoreactive B cells is necessary at the onset of spontaneous systemic autoimmunity in order to expand low-frequency autoreactive B cells. Without such expansion, an encounter with naive cognate autoreactive T cells would be unlikely. However, once expanded, RF B cells (and by extension anti-DNA/RNA B cells) would not need TLR signals to stimulate cognate autoreactive T cells. The fact that initial activation of autoreactive $\mathrm{T}$ cells in vivo was TLR independent in our system likely reflects that we transferred sufficient numbers of cells to make initial T-B interactions possible from the start. Nonetheless, these results show clearly that naive autoreactive B cells can activate unprimed autoreactive $\mathrm{T}$ cells in the absence of a TLR signal.

Conversely, the presence of IC-specific T cells rescued several functional defects of TLR-deficient AM14 B cells. Both proliferation and survival of $7 / r 7 / 9^{-1-}$ B cells were complemented by IC-specific T cells, such that final yields approached WT levels in the case of $1 \mathrm{~B} 9$ and approached about one-third of WT in the case of $13 \mathrm{C} 2$. The inability of the IC-specific T cells to fully restore expansion of $T / r 7 / 9^{-1-} \mathrm{B}$ cells may reflect that only a fraction of AM14 B cells were able to interact with a cognate $\mathrm{T}$ cell in a timely manner after Ag encounter and hence be rescued from the lack of a TLR signal. Alternatively, it could reflect an inferior quality of T cell versus TLR signals in this context. The fact that AM14 B cell proliferation was completely restored in the presence of $13 \mathrm{C} 2$ $\mathrm{T}$ cells in vitro supports the former possibility. Furthermore, 1B9 T cells significantly enhanced differentiation of PBs; however, TLR signaling was still required for the development of IgG2a AFCs.

Collectively, these data suggest a positive feedback model in which small numbers of autoreactive B cells are autonomously activated by engagement of their BCR and TLRs by self-Ag; this expanded population of B cells can present highly concentrated self-peptides to rare and low-affinity T cells in the periphery. Once activated, autoreactive $\mathrm{T}$ cells can provide help, resulting in the TLR-independent activation and proliferation of naive autoreactive $B$ cells and enhancement of the differentiation and expansion of those already activated; however, TLR signaling may play a unique role in isotype switching. 
The ability of IC-specific T cells to induce GC differentiation of AM14 B cells may be reflective of events in later stages of disease. We found that a threshold number of cognate T cells was needed to elicit GC formation in response to antichromatin ICs; such frequencies of self-reactive T cells are not likely achieved until later phases of disease. These results suggest that ICs normally promote an extrafollicular (EF) PB response because endogenous $\mathrm{T}$ cell help is limiting. Of note, only a minority of activated $\mathrm{T}$ cells became Tfh cells. Thus, in the context of an autoimmune response in which B cells receive joint TLR/BCR stimulation, we directly show that there are diverse $\mathrm{T}$ cell outcomes. This could in turn drive multiple outcomes for B cells as well. The EF PB response is enhanced by Bcl6-expressing Tfh-like (T extrafollicular helper) CD4 cells (37, 38). We found that IC-specific T cells enhanced the EF PB response while also promoting GC differentiation. This heterogeneity of both $\mathrm{B}$ and $\mathrm{T}$ cells was striking since these experiments were performed with clonal cell populations. It suggests that cell fate decisions are in part determined stochastically.

The specific need for TLR7, but not TLR9, in AM14 GC differentiation mirrors reports in other systems (39-41). The connection between TLR7 and GC cells is interesting because B cells with RNA-related specificities appear more $\mathrm{T}$ cell dependent. For example, in MRL.Fas ${ }^{\text {lpr }}$ mice in which B cells lack MHC class II, and hence are unable to present antigen to CD4 T cells, the most affected auto Ab was anti-Sm (13). In both patients and lupus-prone mice, RNA-related autoAbs were not affected by B cell depletion $(42,43)$. Such treatment does not deplete long-lived plasma cells, which derive from GC cells, suggesting that B cells with RNA-related specificities derive from GC cells (44). In agreement, AM14 Tlr $9^{-1-}$ B cells stimulated in vitro with PL2-3, which signals through TLR7, are more dependent on $\mathrm{T}$ help than are $T l \mathrm{r} 7^{-/-} \mathrm{B}$ cells.

In the larger picture, the approach we have described is valuable in allowing us to clone self-reactive $\mathrm{T}$ cells from a polyclonal repertoire without any genetic manipulation and without having to choose a candidate Ag. This permitted us to work with authentic self-reactive clones and not (as is often done) TCR Tg mice with specificity for nominal Ags, such as ovalbumin, that are then engineered to be part of "self." The use of natural autoAgs allowed us to evaluate the requirement of endogenous TLR ligands in autoreactive B cell activation and discover that $\mathrm{T}$ cell help can bypass the need for TLR stimulation to a substantial degree. We further were able to determine the natural fate of self-reactive $\mathrm{T}$ cells, showing that they were positively selected and not skewed to a Treg pathway. By studying more than one clone, we found divergent behavior in some functional settings that points to considerable natural heterogeneity. In the future, this method will allow us to further characterize the natural autoreactive $\mathrm{T}$ cell repertoire and greatly expand our currently narrow view of $\mathrm{T}$ cell autoreactive specificities and functions. Furthermore, with these T cells in hand, the next important but challenging task will be to identify the specific proteins from which the stimulatory peptides are derived.

\section{Methods}

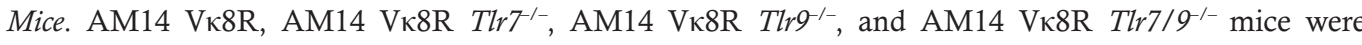

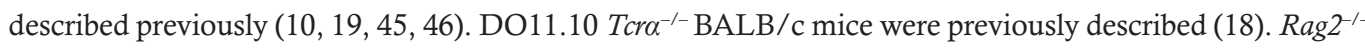
$\mathrm{BALB} / \mathrm{c}$ mice were from Jackson Laboratories.

Cell culture. Primary splenic B cells and T cells were enriched by negative selection according to the manufacturer's instructions (Stemcell Technologies). Total SP APCs were prepared by depleting T cells with anti-Thy1.2 Ab (30H12) and rabbit complement (CedarLane) and then them irradiating with 2,000 cGy. Abs for in vitro stimulation were made in-house as previously described (47). The cell lines were generated in-house or were gifts from Mark Monestier (Temple University, Philadelphia, Pennsylvania).

For cultures detailed in Figure 1B, AM14 B cells and T cells were cultured together at a ratio of 2:1 at $10^{6}$ cells $/ \mathrm{ml}$ in $10 \%$ complete Click's media with BAFF (Peprotech). On the third day, half of the media were replaced with fresh 10\% complete Click's media with BAFF and $10 \mathrm{ng} / \mathrm{ml}$ human IL-2 (Peprotech).

For in vitro stimulation tests with hybridoma T cells, $7.5 \times 10^{5}$ AM14 B cells (or SP APCs) and $5 \times$ $10^{4} \mathrm{~T}$ cells were cultured in 96-well plates in $10 \%$ complete Click's media with BAFF. For in vitro stimulation tests with primary Rg T cells, $3 \times 10^{6} \mathrm{AM} 14 \mathrm{~B}$ cells and $3 \times 10^{5} \mathrm{~T}$ cells were cultured in 12 -well plates in $10 \%$ complete Click's media with BAFF.

Abs for in vitro stimulation. The following Abs were added at $1 \mu \mathrm{g} / \mathrm{ml}$ : Hy1.2, PL2-3, PL2-8, PL9-2, PL9-3, PL9-9, PR1-3, LG4-1, PA1, PA4, 8D8, MRA12, LG2-1, and LG2-2. The following Abs were added at $10 \mu \mathrm{g} / \mathrm{ml}$ : Ha310, BWR4, Y2, and 2G7. The Y2:Sm complexes were formed by mixing 10 $\mu \mathrm{g} / \mathrm{ml}$ of Y2 with purified Sm (Immunovision SMA-3000) at a 1:250 ratio based on the final culture volume. Anti-IgM was used at $15 \mu \mathrm{g} / \mathrm{ml}$. 
Hybridoma fusion. Three days after the third stimulation, cultured T cells were fused with BW5147 cells as previously described (48).

Sequencing TCR genes. RNA was extracted using Qiagen RNeasy mini kits according to the manufacturer's instructions. The RNA was reverse transcribed into cDNA using the iScript kit from Bio-Rad (170-8890). The Tcr $\alpha$ and Tcr $\beta$ genes were amplified as described previously (49). The PCR products were sequenced using constant region primers.

Generation of TCR-encoding RVs. The 13C2 Tcra gene was amplified with forward primer, GCGCCAGATCTACCATGAACACTTCTCCAGCTTTAGTGACTGTG (5'-3'), and reverse primer, CTTCCACGTCTCCTGCTTGCTTTAACAGAGAGAAGTTCGTGGCTCCGGAGCCACTGGACCACAGCCTCAGCGTCATGAG (5'-3'). The 13C2 Trr gene was amplified with forward primer, CTCTGTTAAAGCAAGCAGGAGACGTGGAAGAAAACCCCGGTCCCATGAACAAGTGGGTTTTCTGCTG (5'-3'), and reverse primer, GCGTCGCAATTGGGATCCTCAGGAATTTTTTTTCTTGACCATG (5'-3'). The 1B9 TCR $\alpha$ gene was amplified with forward primer, GCGCCAGATCTACCATGAACTCTTCTCCAGGCTTCATG (5'-3'), and reverse primer, CTTCCACGTCTCCTGCTTGCTTTAACAGAGAGAAGTTCGTGGCTCCGGAGCCACTGGACCACAGCCTCAGCGTCATGAG (5'-3'). The 1B9 TCR $\beta$ gene was amplified with forward primer, GAACTTСТСтСтGTTAAAGCAAGCAGGAGACGTGGAAGAAAACCCCGGTCCCATGGATCCTAGACTTCTTTGCTGTGTG $\left(5^{\prime}-3^{\prime}\right)$, and reverse primer, GCGTCGCAATTGCTCGAGTCAGGAATTTTTTCTCTTGACCATGGC (5'-3').

$R V$ production and infection. Rearranged Tcro and Tcr $\beta$ genes from 13C2 and 1B9 were amplified and cloned into the pMIG II vector (a gift from Dario Vignali, University of Pittsburgh, Pittsburgh, PA) as previously described (24). Platinum-E cells were transfected with pCL-Ecohelper and either 1B9 TCR pMIG II or 13C2 TCR pMIG II plasmids using Lipojet (Signagene). The media were replaced with 10\% Click's media with BAFF for infection of CD4 ${ }^{+} \mathrm{TCR}^{-} 4 \mathrm{G} 4 \mathrm{~T}$ cells (gift from Li Wen, Yale University, New Haven, CT) or $20 \%$ DMEM for bone marrow cells. Platinum-E cells were grown at $32^{\circ} \mathrm{C}$ for RV production. After 18 hours, the RV-containing medium was harvested and added to the cells for infection $\left(\mathrm{CD}^{+}{ }^{+} \mathrm{TCR}^{-} 4 \mathrm{G} 4\right.$ cells or bone marrow cells) with polybrene $(6 \mu \mathrm{g} / \mathrm{ml})$. Cells were spin transduced at $1258 \mathrm{xg}$ at $37^{\circ} \mathrm{C}$ for 90 minutes in 6-well plates. On the following day, a second viral harvest and spinning infection were performed. The next day, the cells rested and were fed with fresh medium.

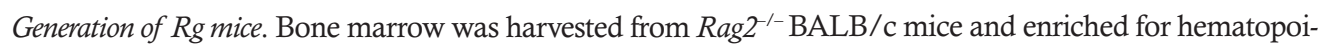
etic progenitor cells using EasySep kits per the manufacturer's instructions (Stemcell Technologies). The cells

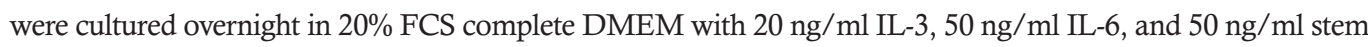
cell factor (Peprotech). Cells were infected with TCR-encoded RV as described below. Five to ten million cells were transferred into DO11.10 Tcro ${ }^{-1-}$ BALB/c mice that had been irradiated 3 hours prior with 4.5 Gy.

Adoptive transfer. Splenic B cells and T cells were enriched by negative selection according to the manufacturer's instructions (Stemcell Technologies). Cells in PBS were injected i.v. at the indicated concentration.

Ascites preparation and immunization. PL2-3 ascites was produced as previously described (50). $200 \mu \mathrm{g}$ was injected i.v. on days 0,2 , and 4 .

Flow cytometry. Staining was performed as previously described (36). PD1 and CXCR5 were stained at room temperature. Live/dead discrimination was determined using Ghost Dye Violet 510 (TONBO). For VPD450 labeling, cells were incubated at $37^{\circ} \mathrm{C}$ for 5 minutes in the dark with $3.5 \mu \mathrm{M}$ VPD450 (BD Biosciences) in PBS.

$\mathrm{Ab}$ clones used for surface staining were as follows: anti-CD19 (1D3, BD Biosciences), anti-CD138 (281-2, BD Biosciences), anti-CD44 (1M7, BD Biosciences), anti-CD38 (90, Biolegend), anti-TCR $\beta$ (H57-597, Biolegend), anti-CD4 (GK1.5, Biolegend), anti-CD8 (TIA 105, Shlomchik laboratory), antiCD62L (Mel-14, Biolegend), anti-CXCR5 (2G8, BD Biosciences), anti-CD95 (Jo2, BD Biosciences), anti-CD25 (PC61, Biolegend), anti-CD3e (145-2C11, Shlomchik laboratory), anti-TCRVß6 (RR4-7, Biolegend), TCRV $\beta 9$ (MR10-2, Biolegend), 4-44 biotin (Shlomchik laboratory), anti-PD1 (RMP1-30, Biolegend), anti-CD162(2PH1, BD Pharmigen), anti-Bc16 (K112-91, BD Biosciences).

Cytokine ELISAs. 96-well Immulon $4 \mathrm{HBX}$ plates were coated overnight at $4^{\circ} \mathrm{C}$ with $1 \mu \mathrm{g} / \mathrm{ml}$ antimouse IL-2 (JES6-1A12, Biolegend) or anti-mouse IFN- $\gamma$ (XMG2.1, Biolegend), blocked for 2 hours with $1 \%$ bovine serum albumin in PBS, and incubated with supernatant overnight at $4^{\circ} \mathrm{C}$. Biotinylated antimouse IL-2 (JES6-5H4, Biolegend) or anti-mouse IFN- $\gamma$ (R4-6A2, Biolegend) was added to the plates and incubated for 2 hours at room temperature, followed by streptavidin-horseradish peroxidase (Southern Biotech) for 1 hour at room temperature. Plates were developed with o-phenylenediamine dihydrochloride. 
4-44 ELISA and ELISpot. ELISA assays were performed similarly to those previously described (45). Biotinylated anti-4-44 was used as the secondary Ab. Plates were developed with o-phenylenediamine dihydrochloride. ELISpot assays were performed as previously described $(18,50)$.

Immunofluorescence. Sections of spleens were prepared and stained as described previously (51). Ab clones used for staining were stained with 4-44-biotin (Shlomchik laboratory), anti-TCR $\beta$-BV421 (H57597, Biolegend), anti-CD45R-Alexa 488 (RA3-6B2, Shlomchik laboratory) or anti-peanut agglutinin (Vector Laboratories), anti-F4/80-Alexa 647 (CI:A3-1, Shlomchik laboratory), and streptavidin-AF555 (Invitrogen). Images were captured on a IX83 fluorescent microscope (Olympus) using a $\times 20$ objective; image analysis was performed using CellSens Dimension software (Olympus).

Statistics. All statistics were calculated using Graphpad Prism software. Each figure lists the statistical test used (1-way, 2-way, or 3-way ANOVAs); multiple hypothesis testing was corrected with Holm-Sidak's.

Study approval. All mice were maintained under specific pathogen-free conditions and handled according to protocols approved by the Institutional Animal Care and Use Committees at Yale University, University of Pittsburgh, and University of Massachusetts School of Medicine.

\section{Author contributions}

JRG and MJS designed research; JRG performed experiments; ATN assisted with experiments; JRG analyzed experiments and wrote the manuscript together with MJS; and AMR provided mice, reagents, and conceptual advice.

\section{Acknowledgments}

We thank the Yale Animal Resource Center and the Division of Laboratory Animal Resources of the University of Pittsburgh for excellent animal care, Jaime Sutherland Cullen and Yujuan Wang for supporting experimental procedures, and Kerstin Nundel for maintaining TLR-deficient mouse colonies and supplying cells. This work was supported by the Lupus Insight Prize and a grant from the Lupus Research Institute to MJS; and by NIH grants 5P01AR050256 (to AMR and MJS) and R01AR044077 (to MJS).

Address correspondence to: Mark J. Shlomchik, 200 Lothrop Street 1052W, Pittsburgh, Pennsylvania 15261, USA. Phone: 412.648.8771; E-mail: MSHLOMCH@pitt.edu.

1. Sang A, Zheng YY, Morel L. Contributions of B cells to lupus pathogenesis. Mol Immunol. 2014;62(2):329-338.

2. Shlomchik MJ. Sites and stages of autoreactive B cell activation and regulation. Immunity. 2008;28(1):18-28.

3. Hoffman RW, et al. Human T cell clones reactive against U-small nuclear ribonucleoprotein autoantigens from connective tissue disease patients and healthy individuals. J Immunol. 1993;151(11):6460-6469.

4. Bockenstedt LK, Gee RJ, Mamula MJ. Self-peptides in the initiation of lupus autoimmunity. J Immunol. 1995;154(7):3516-3524.

5. Danke NA, Koelle DM, Yee C, Beheray S, Kwok WW. Autoreactive T cells in healthy individuals. J Immunol. 2004;172(10):5967-5972.

6. Mohan C, Adams S, Stanik V, Datta SK. Nucleosome: a major immunogen for pathogenic autoantibody-inducing T cells of lupus. J Exp Med. 1993;177(5):1367-1381.

7. Ito Y, et al. Detection of T cell responses to a ubiquitous cellular protein in autoimmune disease. Science. 2014;346(6207):363-368.

8. Kattah NH, et al. Tetramers reveal IL-17-secreting CD4+ T cells that are specific for U1-70 in lupus and mixed connective tissue disease. Proc Natl Acad Sci USA. 2015;112(10):3044-3049.

9. Mohan JF, Calderon B, Anderson MS, Unanue ER. Pathogenic CD4+ T cells recognizing an unstable peptide of insulin are directly recruited into islets bypassing local lymph nodes. J Exp Med. 2013;210(11):2403-2414.

10. Sweet RA, Christensen SR, Harris ML, Shupe J, Sutherland JL, Shlomchik MJ. A new site-directed transgenic rheumatoid factor mouse model demonstrates extrafollicular class switch and plasmablast formation. Autoimmunity. 2010;43(8):607-618.

11. Avalos AM, Busconi L, Marshak-Rothstein A. Regulation of autoreactive B cell responses to endogenous TLR ligands. Autoimmunity. 2010;43(1):76-83.

12. Avalos AM, Ploegh HL. Early BCR events and antigen capture, processing, and loading on MHC class II on B cells. Front Immunol. 2014;5:92.

13. Giles JR, Kashgarian M, Koni PA, Shlomchik MJ. B cell-specific MHC class II deletion reveals multiple nonredundant roles for B cell antigen presentation in murine lupus. J Immunol. 2015;195(6):2571-2579.

14. Lanzavecchia A. Receptor-mediated antigen uptake and its effect on antigen presentation to class II-restricted T lymphocytes. Annu Rev Immunol. 1990;8:773-793.

15. Malynn BA, Romeo DT, Wortis HH. Antigen-specific B cells efficiently present low doses of antigen for induction of T cell proliferation. J Immunol. 1985;135(2):980-988.

16. Chesnut RW, Colon SM, Grey HM. Antigen presentation by normal B cells, B cell tumors, and macrophages: functional and biochemical comparison. J Immunol. 1982;128(4):1764-1768.

17. Casten LA, Pierce SK. Receptor-mediated B cell antigen processing. Increased antigenicity of a globular protein covalently cou- 
pled to antibodies specific for B cell surface structures. J Immunol. 1988;140(2):404-410.

18. Sweet RA, Ols ML, Cullen JL, Milam AV, Yagita H, Shlomchik MJ. Facultative role for T cells in extrafollicular Toll-like receptor-dependent autoreactive B-cell responses in vivo. Proc Natl Acad Sci USA. 2011;108(19):7932-7937.

19. Nündel K, et al. Cell-intrinsic expression of TLR9 in autoreactive B cells constrains BCR/TLR7-dependent responses. J Immunol. 2015;194(6):2504-2512.

20. Lau CM, et al. RNA-associated autoantigens activate B cells by combined B cell antigen receptor/Toll-like receptor 7 engagement. JExp Med. 2005;202(9):1171-1177.

21. Rifkin IR, et al. Immune complexes present in the sera of autoimmune mice activate rheumatoid factor B cells. J Immunol. 2000;165(3):1626-1633.

22. Beaudette-Zlatanova BC, Ling T, Shlomchik MJ, Marshak-Rothstein A, Rifkin IR. B cells and dendritic cells from V kappa 8 light chain transgenic mice activate MRL-lpr/gld CD4+ T cells. J Immunol. 2006;177(1):45-52.

23. Nickerson KM, et al. TLR9 regulates TLR7- and MyD88-dependent autoantibody production and disease in a murine model of lupus. J Immunol. 2010;184(4):1840-1848.

24. Holst J, Szymczak-Workman AL, Vignali KM, Burton AR, Workman CJ, Vignali DA. Generation of T-cell receptor retrogenic mice. Nat Protoc. 2006;1(1):406-417.

25. Herlands RA, Christensen SR, Sweet RA, Hershberg U, Shlomchik MJ. T cell-independent and toll-like receptor-dependent antigen-driven activation of autoreactive B cells. Immunity. 2008;29(2):249-260.

26. Leadbetter EA, Rifkin IR, Hohlbaum AM, Beaudette BC, Shlomchik MJ, Marshak-Rothstein A. Chromatin-IgG complexes activate B cells by dual engagement of IgM and Toll-like receptors. Nature. 2002;416(6881):603-607.

27. William J, Euler C, Christensen S, Shlomchik MJ. Evolution of autoantibody responses via somatic hypermutation outside of germinal centers. Science. 2002;297(5589):2066-2070.

28. William J, Euler C, Leadbetter E, Marshak-Rothstein A, Shlomchik MJ. Visualizing the onset and evolution of an autoantibody response in systemic autoimmunity. J Immunol. 2005;174(11):6872-6878.

29. Mason K, Denney DW, McConnell HM. Myelin basic protein peptide complexes with the class II MHC molecules I-Au and I-Ak form and dissociate rapidly at neutral pH. J Immunol. 1995;154(10):5216-5227.

30. Stadinski BD, Zhang L, Crawford F, Marrack P, Eisenbarth GS, Kappler JW. Diabetogenic T cells recognize insulin bound to IAg7 in an unexpected, weakly binding register. Proc Natl Acad Sci USA. 2010;107(24):10978-10983.

31. Liu GY, Fairchild PJ, Smith RM, Prowle JR, Kioussis D, Wraith DC. Low avidity recognition of self-antigen by T cells permits escape from central tolerance. Immunity. 1995;3(4):407-415

32. Klein L, Klugmann M, Nave KA, Tuohy VK, Kyewski B. Shaping of the autoreactive T-cell repertoire by a splice variant of self protein expressed in thymic epithelial cells. Nat Med. 2000;6(1):56-61.

33. Anderson AC, Nicholson LB, Legge KL, Turchin V, Zaghouani H, Kuchroo VK. High frequency of autoreactive myelin proteolipid protein-specific T cells in the periphery of naive mice: mechanisms of selection of the self-reactive repertoire. $J$ Exp Med. 2000;191(5):761-770.

34. Yan J, Harvey BP, Gee RJ, Shlomchik MJ, Mamula MJ. B cells drive early T cell autoimmunity in vivo prior to dendritic cell-mediated autoantigen presentation. J Immunol. 2006;177(7):4481-4487.

35. Simitsek PD, Campbell DG, Lanzavecchia A, Fairweather N, Watts C. Modulation of antigen processing by bound antibodies can boost or suppress class II major histocompatibility complex presentation of different T cell determinants. J Exp Med. 1995;181(6):1957-1963.

36. Teichmann LL, Schenten D, Medzhitov R, Kashgarian M, Shlomchik MJ. Signals via the adaptor MyD88 in B cells and DCs make distinct and synergistic contributions to immune activation and tissue damage in lupus. Immunity. 2013;38(3):528-540.

37. Odegard JM, et al. ICOS-dependent extrafollicular helper T cells elicit IgG production via IL-21 in systemic autoimmunity. $J$ Exp Med. 2008;205(12):2873-2886.

38. Lee SK, et al. B cell priming for extrafollicular antibody responses requires Bcl-6 expression by T cells. J Exp Med. 2011;208(7):1377-1388.

39. Jackson SW, et al. Opposing impact of B cell-intrinsic TLR7 and TLR9 signals on autoantibody repertoire and systemic inflammation. J Immunol. 2014;192(10):4525-4532.

40. Soni C, et al. B cell-intrinsic TLR7 signaling is essential for the development of spontaneous germinal centers. J Immunol. 2014;193(9):4400-4414.

41. Browne EP. Toll-like receptor 7 controls the anti-retroviral germinal center response. PLoS Pathog. 2011;7(10):e1002293.

42. Pisetsky DS, Grammer AC, Ning TC, Lipsky PE. Are autoantibodies the targets of B-cell-directed therapy? Nat Rev Rheumatol. 2011;7(9):551-556.

43. Ahuja A, Shupe J, Dunn R, Kashgarian M, Kehry MR, Shlomchik MJ. Depletion of B cells in murine lupus: efficacy and resistance. J Immunol. 2007;179(5):3351-3361.

44. Ahuja A, Anderson SM, Khalil A, Shlomchik MJ. Maintenance of the plasma cell pool is independent of memory B cells. Proc Natl Acad Sci USA. 2008;105(12):4802-4807.

45. Nündel K, Busto P, Debatis M, Marshak-Rothstein A. The role of Bruton's tyrosine kinase in the development and BCR/ TLR-dependent activation of AM14 rheumatoid factor B cells. J Leukoc Biol. 2013;94(5):865-875.

46. Prak EL, Weigert M. Light chain replacement: a new model for antibody gene rearrangement. J Exp Med. 1995;182(2):541-548.

47. Shlomchik MJ, Zharhary D, Saunders T, Camper SA, Weigert MG. A rheumatoid factor transgenic mouse model of autoantibody regulation. Int Immunol. 1993;5(10):1329-1341.

48. Kruisbeek AM. Production of Mouse T Cell Hybridomas. In Current Protocols in Immunology. Hoboken, NJ; John Wiley \& Sons: 2001

49. Currier JR, Robinson MA. Spectratype/immunoscope analysis of the expressed TCR repertoire. Curr Protoc Immunol. 2001; Chapter 10:Unit 10.28.

50. Herlands RA, William J, Hershberg U, Shlomchik MJ. Anti-chromatin antibodies drive in vivo antigen-specific activation and somatic hypermutation of rheumatoid factor B cells at extrafollicular sites. Eur J Immunol. 2007;37(12):3339-3351.

51. Hannum LG, Haberman AM, Anderson SM, Shlomchik MJ. Germinal center initiation, variable gene region hypermutation, and mutant B cell selection without detectable immune complexes on follicular dendritic cells. J Exp Med. 2000;192(7):931-942. 